\title{
Complex Analytic Construction of the Kuranishi Family on a Normal Strongly Pseudo-Convex Manifold. II
}

\author{
By \\ Takao Akahori* and Kimio Miyajima**
}

\section{Introduction}

This paper is a continuation of the previous paper [2] by the first named author. The deformation of partially complex structures on a compact strongly pseudo-convex manifold was investigated first by M. Kuranishi [4] in order to give a new insight into the problem of deformations of isolated singularities, and he constructed a family of partially complex structures on a compact strongly pseudo-convex real hypersurface of a complex analytic space with an isolated singularity parametrized by a finite number of parameters and inducing the versal family of deformations of the isolated singularity in some sense. If $(V, x)$ is an analytic subset of a domain in a complex euclidean space with an isolated singular point $x$, we obtain a real submanifold $M$ by cutting the analytic set by a sphere of sufficiently small radius centered at $x$, and $V$ defines a subbundle ${ }^{\circ} T^{\prime \prime}$ of $C T M$, which is called the partially complex structure on $M$ induced from $V$, consisting of all tangent vectors of type $(0,1)$ in $\mathbb{C} T V_{\mid M}$. Kuranishi represented an almost partially complex structure on $M$ of finite distance from ${ }^{\circ} T^{\prime \prime}$ by an element of $\Gamma\left(M, T^{\prime} \otimes\left({ }^{\circ} T^{\prime \prime}\right)^{*}\right)$ and constructed the above-mentioned family of integrable almost partially complex structures making use of the harmonic theory for $\bar{\delta}_{b}$. He tided over the difficulties arising from the non-ellipticity of $\bar{\partial}_{b}$ by making use of Nash-Moser's inverse mapping theorem, thereby the family has only a differentiable structure of class $C^{\infty}$. Then the problem as to when the family may have a complex analytic structure was left open. The first named author answered this problem affirmatively in

Communicated by S. Nakano, February 6, 1979. Revised July 12, 1979.

* Department of Mathematics, University of the Ryukyus, Okinawa 901-24, Japan.

** Department of Mathematics, College of Leberal Arts, Kagoshima University, Kagoshima 890 , Japan. 
[2] in the case that $M$ is normal, $\operatorname{dim}_{\boldsymbol{R}} M \geqq 7$, and $H_{T^{\prime}}^{(2)}=0$, depending on the following ideas: On a compact normal strongly pseudo-convex manifold $M$ there exists a differential complex,

$$
0 \rightarrow \Gamma\left(M,{ }^{o} \bar{T}^{\prime \prime}\right) \stackrel{D_{0}}{\longrightarrow} \Gamma\left(M,{ }^{o} \bar{T}^{\prime \prime} \otimes\left({ }^{o} T^{\prime \prime}\right)^{*}\right) \stackrel{D_{1}}{\longrightarrow} \Gamma\left(M,{ }^{o} \bar{T}^{\prime \prime} \otimes \wedge^{2}\left({ }^{o} T^{\prime \prime}\right)^{*}\right) \rightarrow \cdots,
$$

and the solutions of integrability condition $P(\phi)=0$ can be looked for in $\Gamma(M$, $\left.{ }^{o} \bar{T}^{\prime \prime} \otimes\left({ }^{o} T^{\prime \prime}\right)^{*}\right)$ instead of in $\Gamma\left(M, T^{\prime} \otimes\left({ }^{o} T^{\prime \prime}\right)^{*}\right)$. He avoided the difficulties coming from the non-ellipticity of $\bar{\partial}_{b}$ by introducing the norms \|\|$_{(m)}^{\prime}$ and \|\|$_{(m)}^{\prime \prime}$.

In this paper, following these ideas, we prove Main Theorem by the method used in [5].

Main Theorem. If $M$ is a compact normal strongly pseudo-convex real hypersurface of a complex manifold $N$ with $\operatorname{dim}_{\boldsymbol{R}} M \geqq 7$, there exists a complex analytic family $\{\phi(t) \mid t \in T\}$ of partially complex structures on $M$ of class $C^{2}$ such that $\phi(o)=0$, which is versal at o (cf. Definition 1.6), and the linear term of $\phi(t)$ with respect to the parameter $t$ determines an injective map of the Zariski tangent space $T_{o}^{\prime} T$ of $T$ at o into $H_{\bar{\partial}_{b}}^{1}\left(T^{\prime}\right)$, where $H_{\bar{\partial}_{b}}^{1}\left(T^{\prime}\right)$ denotes the first cohomology group of the complex $\left.\left(\Gamma\left(M, T^{\prime} \otimes \wedge^{q}\left({ }^{o} T^{\prime \prime}\right)\right)^{*}\right), \overline{\widehat{C}}_{T^{\prime}}^{(q)}\right)$.

In Section 1 we recall some notations and results in [1] and [2] which is needed in this paper and give the formulation of Main Theorem. The proof of Main Theorem is given in Sections 2 and 3. In Section 2 we construct a family of partially complex structures on $M$. We prove that the family is the versal family in the sense of M. Kuranishi in Section 3. Throughout this paper, as a parameter space of a family of partially complex structures, we consider only a reduced complex analytic space.

\section{$\S 1 . \quad$ Preliminaries}

In this section, we recall some formulations, notations and results in [1] and [2] which will be needed in Sections 2 and 3.

Let $M$ be a differentiable manifold.

Definition 1.1. By an almost partially complex structure on $M$, we mean a complex subbundle $E$ of $C T M$ of class $C^{\infty}$ such that $E \cap \bar{E}=\{0\}$ where $\bar{E}$ denotes the complex conjugate of $E$.

Further if it satisfies the condition that $[X, Y]$ is in $\Gamma(M, E)$ for any $X, Y$ 
in $\Gamma(M, E)$, we call $E$ a partially complex structure on $M$, where $\Gamma(M, E)$ denotes the vector space of all sections of $E$ over $M$ of class $C^{\infty}$. $\quad M$ with a partially complex structure $E$ is called a partially complex manifold.

If $M$ is a real hypersurface of a complex manifold $N, N$ defines a partially complex structure ${ }^{\circ} T^{\prime \prime}$ on $M$ by

$$
{ }^{\circ} T^{\prime \prime}=T^{\prime \prime} N_{\mid M} \cap \mathbb{C T M}
$$

where $T^{\prime \prime} N$ denotes the complex tangent bundle of $N$ of type $(0,1)$. We call the partially complex structure on $M$ the partially complex structure on $M$ induced from the complex structure of $N$.

On the partially complex manifold $\left(M,{ }^{\circ} T^{\prime \prime}\right)$, we have the (tangential) Cauchy-Riemann operator (cf. [1] $\S 3$ )

$$
\bar{\partial}_{b}: \Gamma\left(U, \wedge^{q}\left({ }^{o} T^{\prime \prime}\right)^{*}\right) \rightarrow \Gamma\left(U, \wedge^{q+1}\left({ }^{o} T^{\prime \prime}\right)^{*}\right)
$$

given by

$$
\begin{aligned}
\bar{\partial}_{b} \phi\left(X_{1}, \ldots, X_{q+1}\right)= & \sum_{i=1}^{q+1}(-1)^{i+1} X_{i} \phi\left(X_{1}, \ldots, \hat{X}_{i}, \ldots, X_{q+1}\right) \\
& +\sum_{i<j}(-1)^{i+j} \phi\left(\left[X_{i}, X_{j}\right], X_{1}, \ldots, \hat{X}_{i}, \ldots, \hat{X}_{j}, \ldots, X_{q+1}\right)
\end{aligned}
$$

for $\phi \in \Gamma\left(U, \wedge^{q}\left({ }^{o} T^{\prime \prime}\right)^{*}\right)$ and $X_{1}, \ldots, X_{q+1} \in \Gamma\left(U,{ }^{o} T^{\prime \prime}\right)$, where $U$ is an open domain of $M$.

We say that a partially complex manifold $\left(M,{ }^{o} T^{\prime \prime}\right)$ with $\operatorname{dim}_{R} M=2 n-1$ is strongly pseudo-convex if ${ }^{\circ} T^{\prime \prime}$ is of rank $n-1$ and its Levi form is positive definite at each point of $M$ (cf. [2], (1.2)).

Definition 1.2. A strongly pseudo-convex manifold $\left(M,{ }^{\circ} T^{\prime \prime}\right)$ is called a normal strongly pseudo-convex manifold if there exists a non-vanishing global real $C^{\infty}$-vector field $\xi$ on $M$ such that

$$
\left[\xi, \Gamma\left(M,{ }^{o} T^{\prime \prime}\right)\right] \subset \Gamma\left(M,{ }^{o} T^{\prime \prime}\right)
$$

and $\xi_{p} \notin \operatorname{Re}\left({ }^{o} T_{p}^{\prime \prime} \oplus^{o} \bar{T}_{p}^{\prime \prime}\right)$ for any $p \in M$, where $\operatorname{Re}\left({ }^{o} T_{p}^{\prime \prime} \oplus{ }^{o} \bar{T}_{p}^{\prime \prime}\right)$ denotes the real part of ${ }^{o} T_{p}^{\prime \prime} \oplus{ }^{o} \bar{T}_{p}^{\prime \prime}$.

From now on, we assume that $M$ is a normal strongly pseudo-convex real hypersurface of a complex manifold $N$ with a fixed real $C^{\infty}$-vector field $\xi$ as above and $\operatorname{dim}_{\boldsymbol{R}} M=2 n-1$.

Then we have the following canonical $C^{\infty}$-splitting of CTM as differentiable vector bundles,

$$
C T M={ }^{\circ} T^{\prime \prime} \oplus{ }^{o} \bar{T}^{\prime \prime} \oplus F
$$


where $F=\boldsymbol{C} \xi$. We set $T^{\prime}={ }^{o} \bar{T}^{\prime \prime} \oplus F$, then there exist the following differential complexes arising from the (tangential) Cauchy-Riemann complex for scalar valued forms,

$$
\begin{aligned}
& 0 \rightarrow \Gamma\left(M, T^{\prime}\right) \stackrel{\bar{\theta}_{T}^{(0)}}{\longrightarrow} \Gamma\left(M, T^{\prime} \otimes\left({ }^{o} T^{\prime \prime}\right)^{*}\right) \stackrel{\overline{\hat{o}}_{T}^{(1)}}{\longrightarrow} \Gamma\left(M, T^{\prime} \otimes \wedge^{2}\left({ }^{o} T^{\prime \prime}\right)^{*}\right) \rightarrow, \\
& 0 \rightarrow \Gamma\left(M,{ }^{o} \bar{T}^{\prime \prime}\right) \stackrel{D_{0}}{\longrightarrow} \Gamma\left(M,{ }^{o} \bar{T}^{\prime \prime} \otimes\left({ }^{o} T^{\prime \prime}\right)^{*}\right) \stackrel{D_{1}}{\longrightarrow} \Gamma\left(M,{ }^{o} \bar{T}^{\prime \prime} \otimes \wedge^{2}\left({ }^{o} T^{\prime \prime}\right)^{*}\right) \rightarrow, \\
& 0 \rightarrow \Gamma(M, F) \stackrel{\bar{\partial}_{F}^{(0)}}{\longrightarrow} \Gamma\left(M, F \otimes\left({ }^{o} T^{\prime \prime}\right)^{*}\right) \stackrel{\bar{\partial}_{F}^{(1)}}{\longrightarrow} \Gamma\left(M, F \otimes \wedge^{2}\left({ }^{o} T^{\prime \prime}\right)^{*}\right) \rightarrow,
\end{aligned}
$$

(cf. [1] and [2], for details).

We define operators which will be needed in Sections 2 and 3, and recall a lemma concerning with them (cf. [2], Lemma 4.2).

For each $q=0,1, \ldots$,

$$
L_{q}: \Gamma\left(M,{ }^{o} \bar{T}^{\prime \prime} \otimes \wedge^{q}\left({ }^{o} T^{\prime \prime}\right)^{*}\right) \rightarrow \Gamma\left(M, F \otimes \wedge^{q+1}\left({ }^{o} T^{\prime \prime}\right)^{*}\right)
$$

is given by

$$
L_{q}(\theta)\left(X_{1}, \ldots, X_{q+1}\right)=\left(\bar{\partial}_{T^{\prime}}^{(q)} \theta\left(X_{1}, \ldots, X_{q+1}\right)\right)_{F}
$$

for $\theta \in \Gamma\left(M,{ }^{o} \bar{T}^{\prime \prime} \otimes \wedge^{q}\left({ }^{o} T^{\prime \prime}\right)^{*}\right)$ and $X_{1}, \ldots, X_{q+1} \in \Gamma\left(M,{ }^{o} T^{\prime \prime}\right)$, where ()$_{F}$ denotes the projection to $F$ according to the splitting $T^{\prime}={ }^{o} \bar{T}^{\prime \prime} \oplus F$.

We note that $L_{q}$ are linear over $C^{\infty}(M)$, the ring of all $C^{\infty}$-functions on $M$, and $L_{0}$ induces a $C^{\infty}$-bundle isomorphism of ${ }^{o} \bar{T}^{\prime \prime}$ to $F \otimes\left({ }^{o} T^{\prime \prime}\right)^{*}$. We denote $L_{1}$ by $L$ according to [2].

Proposition 1.1. (1) $L D_{0}=\bar{\partial}_{F}^{(1)} L_{0}$, (2) $\quad L_{2} D_{1}=\bar{\partial}_{F}^{(2)} L$.

Next, we introduce an hermitian metric on $M$, then hermitian metrics along fibers on ${ }^{\circ} \bar{T}^{\prime \prime}, F$ and $T^{\prime}$ are introduced naturally, and we can speak of the harmonic theory on $\Gamma\left(M,{ }^{o} \bar{T}^{\prime \prime} \otimes \wedge^{q}\left({ }^{o} T^{\prime \prime}\right)^{*}\right), \Gamma\left(M, F \otimes \wedge^{q}\left({ }^{o} T^{\prime \prime}\right)^{*}\right)$ and $\Gamma(M$, $\left.T^{\prime} \otimes \wedge^{q}\left({ }^{\circ} T^{\prime \prime}\right)^{*}\right)$ for $q=1,2, \ldots, n-2$, by J. J. Kohn (cf. [3] Ch. V and [6] $\S 6$ ). In particular, if we denote by $D_{q}^{*}$ (resp. $\square$ ) the adjoint operator of $D_{q}$ with respect to the above hermitian metric (resp. the Laplacian operator $D_{q}^{*} D_{q}$ $\left.+D_{q-1} D_{q-1}^{*}\right)$ and by $H_{o T^{\prime \prime}}^{(q)}$ the vector space of all harmonic elements of $\Gamma(M$, $\left.{ }^{o} \bar{T}^{\prime \prime} \otimes \wedge^{q}\left({ }^{o} T^{\prime \prime}\right)^{*}\right), \quad H_{o}^{(q)} \bar{T}^{\prime \prime}$ is finite dimensional and there exist the Neumann operator

$$
N: \Gamma\left(M,{ }^{o} \bar{T}^{\prime \prime} \otimes \wedge^{q}\left({ }^{o} T^{\prime \prime}\right)^{*}\right) \rightarrow \Gamma\left(M,{ }^{o} \bar{T}^{\prime \prime} \otimes \wedge^{q}\left({ }^{o} T^{\prime \prime}\right)^{*}\right)
$$

and the harmonic operator

$$
\boldsymbol{H}_{o T^{\prime \prime}}^{q}: \Gamma\left(M,{ }^{o} \bar{T}^{\prime \prime} \otimes \wedge^{q}\left({ }^{o} T^{\prime \prime}\right)^{*}\right) \rightarrow H_{o}^{(q)} \bar{T}^{\prime \prime}
$$


with the relations

$$
I=\mathbf{H}_{{ }^{q}}^{q} \bar{T}^{\prime \prime}+\square N \quad \text { and } \quad \square N=N \square,
$$

where $I$ denotes the identity operator of $\Gamma\left(M,{ }^{o} \bar{T}^{\prime \prime} \otimes \wedge^{q}\left({ }^{o} T^{\prime \prime}\right)^{*}\right)$.

Further, on $\Gamma\left(M, F \otimes \wedge^{\prime}\left({ }^{o} T^{\prime \prime}\right)^{*}\right)$ and $\Gamma\left(M, T^{\prime} \otimes \wedge^{q}\left({ }^{o} T^{\prime \prime}\right)^{*}\right)$, we employ similar notations and obtain similar results.

For each non-negative integer $m$ and any open domain $U$ of $M$, we define the Sobolev norm \|\|$_{(m)}$ and the norms \|\|$_{(m)}^{\prime}$, \|\|$_{(m)}^{\prime \prime}$ on $C_{0}^{\infty}(U)$ and then on the spaces $\Gamma_{c}\left(U,{ }^{o} \bar{T}^{\prime \prime} \otimes \wedge^{q}\left({ }^{o} T^{\prime \prime}\right)^{*}\right), \Gamma_{c}\left(U, T^{\prime} \otimes \wedge^{q}\left({ }^{o} T^{\prime \prime}\right)^{*}\right), \Gamma_{c}\left(U, F \otimes \wedge^{q}\left({ }^{o} T^{\prime \prime}\right)^{*}\right)$, where $C_{0}^{\infty}(U)$ (resp. $\Gamma_{c}(U, E)$ ) denotes the vector space of all $C^{\infty}$-functions (resp. all $C^{\infty}$-sections of the vector bundle $E$ ) with compact supports in $U$. (Cf. [2], $\S 3$ for details.) We denote by $\Gamma_{(m)}\left(M,{ }^{o} \bar{T}^{\prime \prime} \otimes \wedge^{q}\left({ }^{o} T^{\prime \prime}\right)^{*}\right), \Gamma_{(m)}^{\prime}(M$, $\left.{ }^{o} \bar{T}^{\prime \prime} \otimes \wedge^{q}\left({ }^{o} T^{\prime \prime}\right)^{*}\right)$ and $\Gamma_{(m)}^{\prime \prime}\left(M,{ }^{o} \bar{T}^{\prime \prime} \otimes \wedge^{q}\left({ }^{o} T^{\prime \prime}\right)^{*}\right)$ the Hilbert spaces obtained by completing $\Gamma\left(M,{ }^{o} \bar{T}^{\prime \prime} \otimes \wedge^{q}\left({ }^{o} T^{\prime \prime}\right)^{*}\right)$ with respect to the norms \|\|$_{(m)},\|\|_{(m)}^{\prime}$ and \|\|$_{(m)}^{\prime \prime}$ respectively, and so on.

We remark that these norms have the following properties: For any open set $U$ of $M$ and each $m \geqq n+1$, there exist constants $c_{m}, c_{m}^{\prime}$ and $d_{m}$ such that

$$
\begin{array}{lll}
\|\phi \psi\|_{(m)} \leqq c_{m}\|\phi\|_{(m)}\|\psi\|_{(m)} & \text { for any } & \phi, \psi \in C_{0}^{\infty}(U), \\
\|\phi \psi\|_{(m)}^{\prime} \leqq c_{m}^{\prime}\|\phi\|_{(m)}^{\prime}\|\psi\|_{(m)}^{\prime} & \text { for any } & \phi, \psi \in C_{0}^{\infty}(U), \\
\left\|\bar{\partial}_{b} \phi\right\|_{(m)} \leqq d_{m}\|\phi\|_{(m)}^{\prime} & \text { for any } & \phi \in C_{0}^{\infty}(U) .
\end{array}
$$

Moreover, we have the following results (cf. [2], Proposition 3.13 and (4.4)).

Proposition 1.2. The following estimates hold.

$$
\begin{aligned}
& \left\|N D_{1}^{*} R_{2}(\phi)\right\|_{(m)}^{\prime \prime} \leqslant\|\phi\|_{(m)}^{\prime 2} \quad \text { uniformly for } \phi \in \Gamma\left(M,{ }^{o} \bar{T}^{\prime \prime} \otimes\left({ }^{o} T^{\prime \prime}\right)^{*}\right) \text {, } \\
& \left\|D_{0} L_{0}^{-1} \bar{\partial}_{F}^{(1) *} N_{F} \phi\right\|_{(m)}^{\prime \prime} \lesssim\|\phi\|_{(m)}^{\prime \prime} \quad \text { uniformly for } \quad \phi \in \Gamma\left(M, F \otimes \wedge^{2}\left({ }^{o} T^{\prime \prime}\right)^{*}\right) \text {, }
\end{aligned}
$$

where $R_{2}$ is a differential operator introduced in Proposition 1.4 below, and see [2] Section 3 for the meaning of the symbol $\lesssim$.

Further, since $L$ is linear over $C^{\infty}(M)$, we have

$$
\|L \phi\|_{(m)}^{\prime \prime} \lesssim\|\phi\|_{(m)}^{\prime \prime} \quad \text { uniformly for } \quad \phi \in \Gamma\left(M,{ }^{o} \bar{T}^{\prime \prime} \otimes\left({ }^{o} T^{\prime \prime}\right)^{*}\right) .
$$

Now we return to a family of partially complex structures on $M$.

First, we recall some propositions (cf. [1] Proposition 1.1, Theorem 2.1 and [2] Proposition 1.7.3).

Proposition 1.3. Let $A$ be the set of all almost partially complex struc- 
tures at a finite distance from ${ }^{\circ} T^{\prime \prime}$. Then there is a natural bijection

$$
b: \Gamma\left(M, T^{\prime} \otimes\left({ }^{o} T^{\prime \prime}\right)^{*}\right) \rightarrow A
$$

such that if we write $b(\phi)={ }^{\phi} T^{\prime \prime}$ for $\phi \in \Gamma\left(M, T^{\prime} \otimes\left({ }^{\circ} T^{\prime \prime}\right)^{*}\right)$ then ${ }^{\phi} T^{\prime \prime}=$ $\left\{X+\phi(X) \mid X \in{ }^{o} T^{\prime \prime}\right\}$. (See [1], for " at a finite distance from ${ }^{\circ} T^{\prime \prime \prime}$.)

Proposition 1.4. An almost partially complex structure ${ }^{\phi} T^{\prime \prime}$ is a partially complex structure if and only if it satisfies the following integrability condition;

$$
P(\phi)=\bar{\partial}_{T^{\prime}}^{(1)} \phi+R_{2}(\phi)+R_{3}(\phi)=0
$$

where $R_{2}(\phi)(X, Y)=[\phi(X), \phi(Y)]_{T^{\prime}}-\phi\left([X, \phi(Y)]_{{ } T^{\prime \prime}}+[\phi(X), Y]_{{ } T^{\prime \prime}}\right) \quad$ and $R_{3}(\phi)(X, Y)=-\phi\left([\phi(X), \phi(Y)]_{{ }^{\circ} T^{\prime \prime}}\right)$ for $X, Y \in \Gamma\left(M,{ }^{o} T^{\prime \prime}\right),[,]_{T^{\prime}}\left(\operatorname{resp} .[,]_{{ }^{\prime \prime}}\right)$ denoting the projection of $\left[\right.$, ] to $T^{\prime}$ (resp. ${ }^{\circ} T^{\prime \prime}$ ) according to the above splitting CTM $=T^{\prime} \oplus^{\circ} T^{\prime \prime}$.

Proposition 1.5. For any $\phi \in \Gamma\left(M,{ }^{o} \bar{T}^{\prime \prime} \otimes\left({ }^{o} T^{\prime \prime}\right)^{*}\right), P(\phi)=0$ if and only if $D_{1} \phi+R_{2}(\phi)=0$ and $L \phi=0$.

Let $E$ be a $C^{\infty}$-vector bundle on $M, T$ an analytic subset of a neighbourhood of the origin of $\boldsymbol{C}^{r}$.

Definition 1.3. A family $\{\phi(t) \mid t \in T\}$ of elements of $\Gamma(M, E)$ (resp. $C^{2}(M, E)$ ) is of class $C^{\infty}$ (resp. $C^{2}$ ) if for each $p \in M$ there exists a neighbourhood $U$ of $p$ such that the map

$$
\tilde{\phi}: U \times T \ni(q, t) \rightarrow \phi(t)(q) \in E
$$

is of class $C^{\infty}$ (resp. $C^{2}$ ), where $\phi(t)(q)$ denotes the value of $\phi(t)$ at $q$ in $E$ and $C^{2}(M, E)$ the vector space of all sections of $E$ over $M$ of class $C^{2}$.

Definition 1.4. A $C^{\infty}$-family (resp. $C^{2}$-family) $\{\phi(t) \mid t \in T\}$ of elements of $\Gamma(M, E)$ (resp. $C^{2}(M, E)$ ) depends complex analytically on $t \in T$ if for each fixed $p \in M, \phi(t)(p)$ is complex analytic in $t$.

Using the notions in Definitions 1.3 and 1.4 , we define a complex analytic family of partially complex structures on $M$.

Definition 1.5. By a complex analytic family of partially complex structures on $M$ parametrized by $T$, we mean a $C^{\infty}$-family $\{\phi(t) \mid t \in T\}$ of elements of $\Gamma\left(M, T^{\prime} \otimes\left({ }^{\circ} T^{\prime \prime}\right)^{*}\right)$ depending complex analytically on $t \in T$ and satisfying $P(\phi(t))=0$ for $t \in T$. 
M. Kuranishi treated our family of partially complex structures on $M$ as a family of isolated singularity in [4] and give the definition of the versal family as follows.

Definition 1.6. A complex analytic family $\{\phi(t) \mid t \in T\}$ of partially complex structures on $M$ with $\phi(0)=0$ is versal at $o \in T$ if the family satisfies the following condition; For any neighbourhood $\tilde{N}$ of $M$ in $N$ and any complex analytic family $\pi: \mathscr{N} \rightarrow S$ of deformations of $\tilde{N}$ with $\mathscr{N}_{o}=\tilde{N}$ for $o \in S$, there are a neighbourhood $S^{\prime}$ of $o$ in $S$, an analytic map $\tau: S^{\prime} \rightarrow T$ and a differentiable embedding $F: M \times S^{\prime} \rightarrow \mathcal{N}_{\mid S^{\prime}}$ of class $C^{\infty}$ depending complex analytically on $s \in S^{\prime}$ (i.e. the map $S^{\prime} \ni s \rightarrow F(p, s) \in \mathscr{N}$ is complex analytic for each fixed $p \in M$ ) such that

(1) $\tau(o)=o$,

(2) $\pi \circ F=p_{2}$ where $p_{2}$ denotes the projection of $M \times S^{\prime}$ onto the second factor,

(3) $F_{\mid M \times o}=\mathrm{id}_{M}$,

(4) for any $s \in S^{\prime}, F_{\mid M \times s}$ induces an isomorphism of the partially complex structure $\phi(\tau(s))$ on $M$ to that induced on $M$ from $\mathscr{N}_{s}$ via $F_{\mid M \times s}$.

If $\{\phi(t) \mid t \in T\}$ is a complex analytic family of partially complex structures on $M$,

$$
\bar{\partial}_{T^{\prime}}^{(1)} \phi(t)+R_{2}(\phi(t))+R_{3}(\phi(t))=0 \text { for } t \in T,
$$

by Proposition 1.5. Then for each $\partial / \partial t \in T_{o}^{\prime} T$,

$$
\bar{\partial}_{T}^{(1)} \partial \phi / \partial t(o)=0
$$

hence $\partial \phi / \partial t(o)$ determines an element of $H \overline{\bar{c}}_{b}\left(T^{\prime}\right)$. Then we have a linear map of $T_{o}^{\prime} T$ to $H_{\partial_{b}}^{1}\left(T^{\prime}\right)$ similar to the case of a family of compact complex manifolds (what is called the Kodaira-Spencer map).

Our purpose is to construct a complex analytic family $\{\phi(t) \mid t \in T\}$ of partially complex structures on $M$ with $\phi(o)=0$ for $o \in T$ which is versal at $o \in T$ in the sense of M. Kuranishi and has the property that the linear map as above is an injective map of $T_{o}^{\prime} T$ into $H_{\bar{\partial}_{b}}^{1}\left(T^{\prime}\right)$. In this paper, we construct only a complex analytic family of $C^{2}$-partially complex structures on $M$ having the same properties as above. In fact, we can define a $C^{2}$-partially complex structure on $M$ in the same way as Definition 1.1 , see that it is represented by an element of $C^{2}\left(M, T^{\prime} \otimes\left({ }^{\circ} T^{\prime \prime}\right)^{*}\right)$ satisfying $P(\phi)=0$ similarly to Propositions 1.3 and 1.4, and get the same result as Proposition 1.5. Then 
we define a complex analytic family of $C^{2}$-partially complex structures on $M$ and the versality of a family in the same way as Definitions 1.5 and 1.6, and the linear map of $T_{o}^{\prime} T$ into $H_{\bar{\delta}_{b}}^{1}\left(T^{\prime}\right)$ as above is also defined for this family.

\section{§2. Construction of a Versal Family}

Let $M$ be a compact normal strongly pseudo-convex real hypersurface of a complex manifold $N$ of complex dimension $n \geqq 4$.

In this section, we construct a family $\phi(t)$ of $C^{2}$-sections of ${ }^{o} \bar{T}^{\prime \prime} \otimes\left({ }^{o} T^{\prime \prime}\right)^{* *}$ over $M$ depending complex analytically on $t$ in a neighbourhood $V$ of the origin of $H_{T^{\prime}}^{(1)}$, and a finite number of holomorphic functions $h_{1}(t), \ldots, h_{l}(t)$ on $V$ satisfying the following relations:

(2.0) $\phi(0)=0$ and $h_{\tau}(0)=0$ for $\tau=1, \ldots, l$,

(2.1) $P(\phi(t))=0$ for $t \in T$,

(2.2) the linear term of $\phi(t)$ is cohomologous to $\sum_{\sigma=1}^{q} \beta_{\sigma} t_{\sigma}$, where $q$ $=\operatorname{dim}_{C} H_{T^{\prime}}^{(1)},\left(\beta_{1}, \ldots, \beta_{q}\right)$ is a base of $H_{T^{\prime}}^{(1)},\left(t_{1}, \ldots, t_{q}\right)$ is the system of coordinates of $H_{T^{\prime}}^{(1)}$ associated with the base, and $T$ is an analytic subset of $V$ defined by $h_{1}(t)=\cdots=h_{l}(t)=0$.

For the proof, we first introduce some notations.

For each $\psi \in \Gamma\left(M, T^{\prime} \otimes\left({ }^{o} T^{\prime \prime}\right)^{*}\right)$, we set $\mathscr{L} \psi=\psi-\bar{\partial}_{T^{\prime}}^{(1)} \theta_{\psi}$, where $0_{\psi}$ is a unique element of $\Gamma\left(M,{ }^{o} \bar{T}^{\prime \prime}\right)$ such that

$$
\left[X, \theta_{\psi}\right]_{F}=\psi(X)_{F} \quad \text { for any } \quad X \in \Gamma\left(M,{ }^{o} T^{\prime \prime}\right) .
$$

Then it is easily seen that $\mathscr{L} \psi$ is in $\Gamma\left(M,{ }^{o} \bar{T}^{\prime \prime} \otimes\left({ }^{o} T^{\prime \prime}\right)^{*}\right)$.

Proposition 2.1 ([2] Proposition 5.1). The map $\mathscr{L}_{\mid H_{T \prime}^{(1)}}$ is injective.

Then if we set $\mathscr{H}=\mathscr{L}\left(H_{T^{\prime}}^{(1)}\right), \mathscr{H}$ also represents the first cohomology group $H_{\bar{\partial}_{b}}^{1}\left(T^{\prime}\right)$.

Let $\left(\gamma_{1}^{\prime}, \ldots, \gamma_{p}^{\prime}\right)$ be an orthonormal base of $\boldsymbol{H}_{F}^{2} L\left(H_{{ }_{0}}^{(1)}\right)$ with respect to the $L^{2}$-inner product $($,$) on \Gamma\left(M, F \otimes \wedge^{2}\left({ }^{\circ} T\right)^{*}\right)$ introduced in Section 1 and $\left(\gamma_{1}, \ldots, \gamma_{p}\right)$ elements of $H_{o}^{(1)}$, such that $\boldsymbol{H}_{F}^{2} L\left(\gamma_{i}\right)=\gamma_{i}^{\prime}$ for $i=1, \ldots, p$.

For $\eta \in \Gamma\left(M, F \otimes \wedge^{2}\left({ }^{o} T^{\prime \prime}\right)^{*}\right)$, we set $\rho(\eta)=\sum_{i=1}^{q}\left(\eta, \gamma_{i}^{\prime}\right) \gamma_{i}$.

Then we have the following lemma.

Lemma 2.2. (1) $\boldsymbol{H}_{F}^{2} L \rho \boldsymbol{H}_{F}^{2} L \boldsymbol{H}_{{ }^{1} \bar{T}^{\prime \prime}}^{1}=\boldsymbol{H}_{F}^{2} L \boldsymbol{H}_{{ }^{\prime}}^{1} \bar{T}^{\prime \prime}$,

(2) $\left(L \rho \boldsymbol{H}_{F}^{2}\right)^{2}=L \rho \boldsymbol{H}_{F}^{2}$. 
Proof. (1) Let $\eta \in H_{o}^{(1)}$. Then

$$
\begin{aligned}
\boldsymbol{H}_{F}^{2} L \rho \boldsymbol{H}_{F}^{2} L(\eta) & =\boldsymbol{H}_{F}^{2} L\left(\sum_{i=1}^{p}\left(\boldsymbol{H}_{F}^{2} L(\eta), \gamma_{i}^{\prime}\right) \gamma_{i}\right) \\
& =\sum_{i=1}^{p}\left(\boldsymbol{H}_{F}^{2} L(\eta), \gamma_{i}^{\prime}\right) \gamma_{i}^{\prime} \\
& =\mathbb{H}_{F}^{2} L(\eta) .
\end{aligned}
$$

(2) Let $\psi \in H_{F}^{(2)}$. Then

$$
\begin{aligned}
L \rho \boldsymbol{H}_{F}^{2} L \rho(\psi) & =L \rho \boldsymbol{H}_{F}^{2} L\left(\sum_{i=1}^{p}\left(\psi, \gamma_{i}^{\prime}\right) \gamma_{i}\right) \\
& =L \rho\left(\sum_{i=1}^{p}\left(\psi, \gamma_{i}^{\prime}\right) \gamma_{i}^{\prime}\right) \\
& =\sum_{i=1}^{p}\left(\psi, \gamma_{i}^{\prime}\right) L\left(\gamma_{i}\right)=L \rho(\psi) .
\end{aligned}
$$

In view of Proposition 1.5 and Proposition 2.1, we shall construct $\phi(t)$ and $h_{1}(t), \ldots, h_{l}(t)$ satisfying the following:

$$
\begin{aligned}
& \text { (2.0) } \phi(0)=0 \text { and } h_{\tau}(0)=0 \text { for } \tau=1, \ldots, l \text {, } \\
& \text { (2.1.1) } D_{1} \phi(t)+R_{2}(\phi(t))=0 \text { for } t \in T, \\
& \text { (2.1.2) } L \phi(t)=0 \text { for } t \in T,
\end{aligned}
$$

(2.2) the linear term of $\phi(t)$ is equal to $\sum_{\sigma=1}^{q}\left(\mathscr{L} \beta_{\sigma}\right) t_{\sigma}$.

Before beginning the proof, it is convenient to introduce the following notation: Let $U$ and $D$ be open sets in $M$ and $C^{q}$ respectively, $h_{1}(t), \ldots, h_{l}(t)$ holomorphic functions on $D$, and $E$ a vector bundle on $U$. For two families $\phi(t)$ and $\psi(t)$ of $C^{\infty}$-sections (resp. $C^{2}$-sections) of $E$ on $U$ which depend complex analytically on $t$ in $D$, by

$$
\phi(t) \equiv \psi(t) \quad \bmod \left(h_{1}(t), \ldots, h_{l}(t), t^{\mu}\right),
$$

we mean that there exist families $a_{1}(t), \ldots, a_{l}(t)$ of $C^{\infty}$-sections (resp. $C^{2}$-sections) of $E$ on $U$ which depend complex analytically on $t$ in $D$ such that $\phi(t)-\psi(t)$ $-\sum_{\tau=1}^{l} a_{\tau}(t) h_{\tau}(t)$ contains no term of total degree less than $\mu$ as a power series in $\left(t_{1}, \ldots, t_{q}\right)$.

\section{(I) Construction of Formal Solution}

In this paragraph, we shall construct formal power series $\phi(t), h_{1}(t), \ldots$, $h_{l}(t)$ in $t=\left(t_{1}, \ldots, t_{q}\right)$ satisfying the formal versions of $(2.0),(2.1 .1),(2.1 .2)$ and (2.2) below. To do this, we construct, for each $\mu \geqq 0$, a $\Gamma\left(M,{ }^{o} \bar{T}^{\prime \prime} \otimes\left({ }^{o} T^{\prime \prime}\right)^{*}\right)$ valued polynomial $\phi^{\mu}(t)$ in $t$ of degree $\mu$ and an $H_{T^{\prime}}^{(2)}$-valued polynomial $h^{\mu}(t)$ in $t$ of the same degree, satisfying the following:

$$
\begin{aligned}
& \phi^{0}=0 \quad \text { and } \quad h^{0}=0, \\
& D_{1} \phi^{\mu}(t)+R_{2}\left(\phi^{\mu}(t)\right) \equiv 0 \quad \bmod \left(h^{\mu}(t), t^{\mu+1}\right),
\end{aligned}
$$




$$
\begin{aligned}
& L \phi^{\mu}(t) \equiv 0 \quad \bmod \left(h^{\mu}(t), t^{\mu+1}\right), \\
& \phi^{1}(t)=\sum_{\sigma=1}^{q}\left(\mathscr{L} \beta_{\sigma}\right) t_{\sigma}, \\
& \phi^{\mu-1}(t) \equiv \phi^{\mu}(t) \quad \bmod \left(t^{\mu}\right),
\end{aligned}
$$

and

$$
h^{\mu-1}(t) \equiv h^{\mu}(t) \quad \bmod \left(t^{\mu}\right),
$$

where $h^{\mu}(t)=h_{1}^{\mu}(t) e_{1}+\cdots+h_{l}^{\mu}(t) e_{l}$ for some fixed base $\left(e_{1}, \ldots, e_{l}\right)$ of $H_{T^{\prime}}^{(2)}$ and $\bmod \left(h^{\mu}(t), t^{\mu+1}\right)=\bmod \left(h_{1}^{\mu}(t), \ldots, h_{l}^{\mu}(t), t^{\mu+1}\right)$.

We construct such $\phi^{\mu}(t)$ and $h^{\mu}(t)$ by induction on $\mu$, while from technical reasons, we impose on them the following additional conditions for the induction:

$$
\begin{aligned}
& D_{1}^{*}\left(P\left(\phi^{\mu}(t)\right)_{\sigma_{T^{\prime \prime}}}\right) \equiv 0 \quad \bmod \left(t^{\mu+1}\right), \\
& \bar{\partial}_{F}^{(1) *} L \phi^{\mu}(t)=0, \\
& L \rho \boldsymbol{H}_{F}^{2} L \phi^{\mu}(t)=0 .
\end{aligned}
$$

In view of (2.0) and (2.2), we set

$$
\phi^{0}=0, \quad h^{0}=0,
$$

and

$$
\phi^{1}(t)=\sum_{\sigma=1}^{q}\left(\mathscr{L} \beta_{\sigma}\right) t_{\sigma}, \quad h^{1}(t)=0 .
$$

Then $\phi^{1}(t)$ is $\Gamma\left(M,{ }^{o} \bar{T}^{\prime \prime} \otimes\left({ }^{o} T^{\prime \prime}\right)^{*}\right)$-valued, $(2.3)_{1}$ is clearly satisfied, and since $\bar{\partial}_{T^{\prime}}^{(1)} \phi^{1}(t)=0,(2.1 .1)_{1},(2.1 .2)_{1},(2.5)_{1}$, and (2.6) ${ }_{1}$ are satisfied. Moreover we have $P\left(\phi^{1}(t)\right) \equiv 0 \bmod \left(t^{2}\right)$, so $(2.4)_{1}$ is also satisfied.

Suppose then that, for some $\mu \geqq 2, \phi^{v}(t)$ and $h^{v}(t)$ are already determined for all $v<\mu$ in such a way that $(2.1 .1)_{v},(2.1 .2)_{v},(2.3)_{v}-(2.6)_{v}$ are all satisfied.

Then we set

$$
\phi_{\mu}(t)=-\kappa_{t}^{\mu}\left\{\left(I-D_{0} L_{0}^{-1} \bar{\partial}_{F}^{(1) *} N_{F} L\right)\left(I-\rho H_{F}^{2} L\right) D_{1}^{*} N\left(P\left(\phi^{\mu-1}(t)\right)_{\sigma_{T^{\prime \prime}}}\right)\right\}
$$

and

$$
\phi^{\mu}(t)=\phi^{\mu-1}(t)+\phi_{\mu}(t),
$$

where $\kappa_{t}^{\mu}$ is the operator taking the homogeneous term of total degree $\mu$ in $\left(t_{1}, \ldots, t_{q}\right)$. Since $N$ and $N_{F}$ are $C^{\infty}$-operators, and $\phi^{\mu-1}(t)$ is $\Gamma(M$, $\left.{ }^{o} \bar{T}^{\prime \prime} \otimes\left({ }^{o} T^{\prime \prime}\right)^{*}\right)$-valued by induction, $\phi^{\mu}(t)$ also is $\Gamma\left(M,{ }^{o} \bar{T}^{\prime \prime} \otimes\left({ }^{\circ} T^{\prime \prime}\right)^{*}\right)$-valued.

Next, we define $h^{\mu}(t)$ by the following congruence:

$$
h^{\mu}(t) \equiv \boldsymbol{H}_{T^{\prime}}^{2}\left(P\left(\phi^{\mu-1}(t)\right)\right) \bmod \left(t^{\mu+1}\right) .
$$


Then we shall show that these $\phi^{\mu}(t)$ and $h^{\mu}(t)$ satisfy the desired equalities. (We set $\phi_{1}(t)=\phi^{1}(t)$ in the below.)

$$
\begin{aligned}
& (2.4)_{\mu}: \quad D_{1}^{*}\left(P\left(\phi^{\mu}(t)\right)_{\sigma_{T^{\prime \prime}}}\right) \\
& \equiv D_{1}^{*}\left(P\left(\phi^{\mu-1}(t)\right)_{{ }^{\prime} \bar{T}^{\prime \prime}}+D_{1} \phi_{\mu}(t)\right) \quad \bmod \left(t^{\mu+1}\right) \\
& =D_{1}^{*}\left(P\left(\phi^{\mu-1}(t)\right)_{{ }_{\circ} T^{\prime \prime}}\right)-D_{1}^{*} D_{1} \kappa_{t}^{\mu}\left\{N D_{1}^{*}\left(P\left(\phi^{\mu-1}(t)\right)_{{ }_{o} T^{\prime \prime}}\right)\right\} \\
& =D_{1}^{*}\left(P\left(\phi^{\mu-1}(t)\right)_{{ }_{T^{\prime \prime}}}\right)-\kappa_{t}^{\mu} D_{1}^{*}\left(P\left(\phi^{\mu-1}(t)\right)_{o} \bar{T}^{\prime \prime}\right) \\
& \equiv 0 \quad \bmod \left(t^{\mu+1}\right), \quad\left(\text { by }(2.4)_{\mu-1}\right) \text {. } \\
& (2.5)_{\mu}: \quad \bar{\partial}_{F}^{(1) *} L \phi^{\mu}(t) \\
& =-\kappa_{t}^{\mu}\left\{\bar{\partial}_{F}^{(1) *}\left(L-L D_{0} L_{0}^{-1} \bar{\partial}_{F}^{(1) *} N_{F} L\right)\left(I-\rho \mathbb{H}_{F}^{2} L\right) D_{1}^{*} N\left(P\left(\phi^{\mu-1}(t)\right)_{{ }^{\prime} T^{\prime \prime}}\right)\right\}, \\
& \text { (by } \left.(2.5)_{\mu-1}\right) \text {, } \\
& =-\kappa_{t}^{\mu}\left\{\bar{\partial}_{F}^{(1) *}\left(I-\bar{\partial}_{F}^{(1)} \bar{\partial}_{F}^{(1) *} N_{F}\right) L\left(I-\rho \mathbb{H}_{F}^{2} L\right) D_{1}^{*} N\left(P\left(\phi^{\mu-1}(t)\right)_{o} \bar{T}^{\prime \prime}\right)\right\}, \\
& \text { (by Proposition 1.1), } \\
& =-\kappa_{t}^{\mu}\left\{\bar{\partial}_{F}^{(1) *}\left(\bar{\partial}_{F}^{(2) *} \bar{\partial}_{F}^{(2)} N_{F}+\mathbb{H}_{F}^{2}\right) L\left(I-\rho \mathbb{H}_{F}^{2} L\right) D_{1}^{*} N\left(P\left(\phi^{\mu-1}(t)\right)_{\bar{T}^{\prime \prime}}\right)\right\} \\
& =0 \text {. } \\
& =-\kappa_{t}^{\mu}\left\{L \rho \boldsymbol{H}_{F}^{2}\left(I-\bar{\partial}_{F}^{(1)} \bar{\partial}_{F}^{(1) *} N_{F}\right) L\left(I-\rho \mathbb{H}_{F}^{2} L\right) D_{1}^{*} N\left(P\left(\phi^{\mu-1}(t)\right)_{\circ T^{\prime \prime}}\right)\right\}, \\
& \text { (by }(2.6)_{\mu-1} \text { and Proposition 1.1), } \\
& =-\kappa_{t}^{\mu}\left\{L \rho \mathbb{H}_{F}^{2}\left(\bar{\partial}_{F}^{(2)} * \bar{\partial}_{F}^{(2)} N_{F}+\mathbb{H}_{F}^{2}\right) L\left(I-\rho \mathbb{H}_{F}^{2} L\right) D_{1}^{*} N\left(P\left(\phi^{\mu-1}(t)\right)_{o} \bar{T}^{\prime \prime}\right)\right\} \\
& =-\kappa_{t}^{\mu}\left\{\left(L \rho \boldsymbol{H}_{F}^{2}-\left(L \rho H_{F}^{2}\right)^{2}\right) L D_{1}^{*} N\left(P\left(\phi^{\mu-1}(t)\right)_{\sigma_{T^{\prime \prime}}}\right)\right\} \\
& =0 \text {, (by Lemma 2.2, (2)). }
\end{aligned}
$$

$(2.3)_{\mu}: \quad$ It is clear that

$$
\begin{aligned}
\phi^{\mu-1}(t) & \equiv \phi^{\mu}(t) \quad \bmod \left(t^{\mu}\right) \\
h^{\mu}(t) & \equiv \mathbb{H}_{T^{\prime}}^{2}\left(P\left(\phi^{\mu-1}(t)\right)\right) \quad \bmod \left(t^{\mu+1}\right) \\
& \equiv \mathbb{H}_{T^{\prime}}^{2}\left(P\left(\phi^{\mu-2}(s)\right)+\bar{\partial}_{T^{\prime}}^{(1)} \phi_{\mu-1}(t)\right) \bmod \left(t^{\mu}\right) \\
& \equiv h^{\mu-1}(t) \bmod \left(t^{\mu}\right) .
\end{aligned}
$$

To prove $(2.1 .1)_{\mu}$, we first show the following lemma.

\section{Lemma 2.3.}

$$
\begin{aligned}
& \bar{\partial}_{T^{\prime}}^{(2)} P\left(\phi^{\mu-1}(t)\right) \equiv 0 \quad \bmod \left(h^{\mu}(t), t^{\mu+1}\right) \\
& \mathbb{H}_{o}^{2} \bar{T}^{\prime \prime}\left(P\left(\phi^{\mu-1}(t)\right)_{o} \bar{T}^{\prime \prime}\right) \equiv 0 \quad \bmod \left(h^{\mu}(t), t^{\mu+1}\right), \\
& D_{2}\left(P\left(\phi^{\mu-1}(t)\right)_{o} \bar{T}^{\prime \prime}\right) \equiv 0 \quad \bmod \left(h^{\mu}(t), t^{\mu+1}\right)
\end{aligned}
$$

Proof. $(2.7)_{\mu}$ : By Proposition 4.5 in [4], we have

$$
\bar{\partial}_{T^{\prime}}^{\phi \mu-1}(t) P\left(\phi^{\mu-1}(t)\right)=0 .
$$


Since $P\left(\phi^{\mu-1}(t)\right) \equiv 0 \bmod \left(h^{\mu-1}(t), t^{\mu}\right)$, we infer that $\bar{\partial}_{T^{\prime}}^{(2)} P\left(\phi^{\mu-1}(t)\right) \equiv 0 \bmod$ $\left(t h^{\mu-1}(t), t^{\mu+1}\right)$. Since $t h^{\mu-1}(t) \equiv 0 \bmod \left(h^{\mu}(t), t^{\mu+1}\right)$, by $(2.3)_{\mu},(2.7)_{\mu}$ follows.

$(2.8)_{\mu}$ and $(2.9)_{\mu}$ : If we set $\psi(t)=-\bar{\partial}_{T^{\prime}}^{(1) *} N_{T^{\prime}} P\left(\phi^{\mu-1}(t)\right)$,

$$
\begin{aligned}
\bar{\partial}_{T^{\prime}}^{(1)} \psi(t) & =-\bar{\partial}_{T^{\prime}}^{(1)} \bar{\partial}_{T^{\prime}}^{(1) *} N_{T^{\prime}} P\left(\phi^{\mu-1}(t)\right) \\
& =-P\left(\phi^{\mu-1}(t)\right)+\bar{\partial}_{T^{\prime}}^{(2)} * \bar{\partial}_{T^{\prime}}^{(2)} N_{T^{\prime}} P\left(\phi^{\mu-1}(t)\right)+\boldsymbol{H}_{T^{\prime}}^{2} P\left(\phi^{\mu-1}(t)\right) \\
& =-P\left(\phi^{\mu-1}(t)\right)+N_{T^{\prime}} \bar{\partial}_{T^{\prime}}^{(2) *} \bar{\partial}_{T^{\prime}}^{(2)} P\left(\phi^{\mu-1}(t)\right)+\boldsymbol{H}_{T^{\prime}}^{2} P\left(\phi^{\mu-1}(t)\right) \\
& \equiv-P\left(\phi^{\mu-1}(t)\right) \quad \bmod \left(h^{\mu}(t), t^{\mu+1}\right),
\end{aligned}
$$

(by $(2.7)_{\mu}$ and the definition of $h^{\mu}(t)$ ). Hence, by the definition of $\mathscr{L}$, we have

$$
\bar{\partial}_{T^{\prime}}^{(1)} \mathscr{L} \psi(t)+P\left(\phi^{\mu-1}(t)\right) \equiv 0 \quad \bmod \left(h^{\mu}(t), t^{\mu+1}\right)
$$

and

$$
D_{1} \mathscr{L} \psi(t)+P\left(\phi^{\mu-1}(t)\right)_{{ }^{\prime} \bar{T}^{\prime \prime}} \equiv 0 \bmod \left(h^{\mu}(t), t^{\mu+1}\right) .
$$

From these, we infer $(2.8)_{\mu}$ and $(2.9)_{\mu}$.

$(2.1 .1)_{\mu}: \quad$ By the definition of $\phi_{\mu}(t)$,

$$
\begin{aligned}
D_{1} \phi_{\mu}(t) & =-\kappa_{t}^{\mu}\left\{D_{1} N D_{1}^{*}\left(P\left(\phi^{\mu-1}(t)\right)_{{ }_{o} \bar{T}^{\prime \prime}}\right)\right\} \\
& \equiv-N D_{1} D_{1}^{*}\left(P\left(\phi^{\mu-1}(t)\right)_{{ }^{\circ} T^{\prime \prime}}\right) \bmod \left(t^{\mu+1}\right), \quad\left(\text { by }(2.4)_{\mu-1}\right) .
\end{aligned}
$$

Then

$$
\begin{aligned}
& D_{1} \phi^{\mu}(t)+R_{2}\left(\phi^{\mu}(t)\right) \\
& \quad \equiv D_{1} \phi_{\mu}(t)+P\left(\phi^{\mu-1}(t)\right)_{o_{T^{\prime \prime}}} \quad \bmod \left(t^{\mu+1}\right) \\
& \quad \equiv N D_{2}^{*} D_{2}\left(P\left(\phi^{\mu-1}(t)\right)_{o} \bar{T}^{\prime \prime}\right)+\boldsymbol{H}_{\mathrm{o}_{T^{\prime \prime}}}^{2}\left(P\left(\phi^{\mu-1}(t)\right)_{{ }_{\bar{T}^{\prime \prime}}}\right) \quad \bmod \left(t^{\mu+1}\right) \\
& \quad \equiv 0 \bmod \left(h^{\mu}(t), t^{\mu+1}\right), \quad\left(\text { by }(2.8)_{\mu} \text { and }(2.9)_{\mu}\right) .
\end{aligned}
$$

$(2.1 .2)_{\mu}: \quad L \phi^{\mu}(t)$

$$
\equiv L \phi^{\mu-1}(t)-\left\{\left(I-\bar{\partial}_{F}^{(1)} \bar{\partial}_{F}^{(1)} * N_{F}\right)\left(I-L \rho \boldsymbol{H}_{F}^{2}\right) L D_{1}^{*} N\left(P\left(\phi^{\mu-1}(t)\right)_{{ }^{\prime \prime}}\right)\right\} \bmod \left(t^{\mu+1}\right),
$$

(by $(2.4)_{\mu-1}$ and Proposition 1.1). Now, if we set $\psi(t)=-\bar{\partial}_{T^{\prime}}^{(1) *} N_{T^{\prime}} P\left(\phi^{\mu-1}(t)\right)$, as in the proof of $(2.8)_{\mu}$ and (2.9) $)_{\mu}$, we have

$$
D_{1} \mathscr{L} \psi(t)+P\left(\phi^{\mu-1}(t)\right)_{{ }^{\circ T^{\prime \prime}}}=o(t)_{{ }^{T^{\prime \prime}}}
$$

and

$$
L \mathscr{L} \psi(t)+L \phi^{\mu-1}(t)=o(t)_{F}
$$

where $o(t)=\bar{\partial}_{T^{\prime}}^{(2) *} \bar{\partial}_{T^{\prime}}^{(2)} N_{T^{\prime}} P\left(\phi^{\mu-1}(t)\right)+\boldsymbol{H}_{T}^{2}, P\left(\phi^{\mu-1}(t)\right)$. Then

$$
\begin{aligned}
& L D_{1}^{*} N\left(P\left(\phi^{\mu-1}(t)\right)_{{ }^{\circ} T^{\prime \prime}}\right) \\
& \quad=-L D_{1}^{*} N D_{1} \mathscr{L} \psi(t)+L D_{1}^{*} N\left(o(t)_{{ }_{o} T^{\prime \prime}}\right) \\
& \quad=-L \mathscr{L} \psi(t)+L D_{0} D_{0}^{*} N \mathscr{L} \psi(t)+L \boldsymbol{H}_{{ }^{\prime} T^{\prime \prime}} \mathscr{L} \psi(t)+L D_{1}^{*} N\left(o(t)_{{ }^{o} \bar{T}^{\prime \prime}}\right)
\end{aligned}
$$




$$
=L \phi^{\mu-1}(t)+L D_{0} D_{0}^{*} N \mathscr{L} \psi(t)+L \boldsymbol{H}_{{ }^{1} \bar{T}^{\prime \prime}}^{1} \mathscr{L} \psi(t)+L D_{1}^{*} N\left(o(t)_{o} \bar{T}^{\prime \prime}\right)-o(t)_{F} .
$$

Hence, since

$$
L \phi^{\mu-1}(t)-\left(I-\bar{\partial}_{F}^{(1)} \bar{\partial}_{F}^{(1)} * N_{F}\right)\left(I-L \rho \mathbb{H}_{F}^{2}\right) L \phi^{\mu-1}(t)=0
$$

by $(2.5)_{\mu-1}$ and $(2.6)_{\mu-1}$, we have

$$
\begin{aligned}
L \phi^{\mu}(t) \equiv & -\left(I-\bar{\partial}_{F}^{(1)} \bar{\partial}_{F}^{(1) *} N_{F}\right)\left(I-L \rho \boldsymbol{H}_{F}^{2}\right)\left\{L D_{0} D_{0}^{*} N \mathscr{L} \psi(t)+L \boldsymbol{H}_{o}^{1} T^{\prime \prime} \mathscr{L} \psi(t)\right\} \\
& \quad \bmod \left(h^{\mu}(t), t^{\mu+1}\right), \quad\left(\text { since } o(t) \equiv 0 \bmod \left(h^{\mu}(t), t^{\mu+1}\right) \text { by }(2.7)_{\mu}\right. \text { and } \\
& \text { the definition of } \left.h^{\mu}(t)\right), \\
= & -\left(I-\bar{\partial}_{F}^{(1)} \bar{\partial}_{F}^{(1) *} N_{F}\right) \bar{\partial}_{F}^{(1)} L_{0} D_{0}^{*} N \mathscr{L} \psi(t) \\
& -\left(\bar{\partial}_{F}^{(2) *} \bar{\partial}_{F}^{(2)} N_{F}+\boldsymbol{H}_{F}^{2}\right)\left(I-L \rho \boldsymbol{H}_{F}^{2}\right) L \boldsymbol{H}_{o}^{1} \bar{T}^{\prime \prime} \mathscr{L} \psi(t), \quad \text { (by Proposition 1.1), } \\
= & -\bar{\partial}_{F}^{(2) *} \bar{\partial}_{F}^{(2)} N_{F}\left(I-L \rho \boldsymbol{H}_{F}^{2}\right) L \boldsymbol{H}_{o}^{1} \bar{T}^{\prime \prime} \mathscr{L} \psi(t), \quad(\text { by Lemma 2.2, (1)), } \\
= & -N_{F} \bar{\partial}_{F}^{(2) *} \bar{\partial}_{F}^{(2)} L\left(I-\rho \boldsymbol{H}_{F}^{2} L\right) \boldsymbol{H}_{o}^{1} \bar{T}^{\prime \prime} \mathscr{L} \psi(t) \\
= & -N_{F} \bar{\partial}_{F}^{(2) *} L_{2} D_{1}\left(I-\rho \boldsymbol{H}_{F}^{2} L\right) \boldsymbol{H}_{o}^{1} \bar{T}^{\prime \prime} \mathscr{L} \psi(t), \quad \text { (by Proposition 1.1), } \\
= & 0 .
\end{aligned}
$$

Thus (2.1.2) $)_{\mu}$ is proved.

This completes the inductive constructions of $\phi^{\mu}(t)$ and $h^{\mu}(t)$

\section{(II) Proof of Convergence}

In this paragraph, we shall prove that the formal power series $\phi(t)=$ $\lim _{\mu \rightarrow \infty} \phi^{\mu}(t)$ and $h(t)=\lim _{\mu \rightarrow \infty} h^{\mu}(t)$ in $\left(t_{1}, \ldots, t_{q}\right)$ are convergent with respect to \|\|$_{(m)}^{\prime \prime}$-norm and | |-norm respectively where | | denotes the euclidean norm on the finite dimensional vector space $H_{T^{\prime}}^{(2)}$. To prove this, we first show the existence of a convergent power series $A(t)$ in $\left(t_{1}, \ldots, t_{q}\right)$ with real non-negative coefficients and with the following property:

$$
\left\|\phi^{\mu}(t)\right\|_{(m)}^{\prime \prime} \ll A(t) \quad \text { for all } \quad \mu \geqq 1 .
$$

(Cf. [2], for the meaning of the symbol «.)

First we fix $m \geqq n+2$, and set

$$
A(t)=b / 16 c \sum_{\mu=1}^{\infty}\left(c^{\mu} / \mu^{2}\right)\left(t_{1}+\cdots+t_{q}\right)^{\mu}
$$

where $b$ and $c$ are positive numbers to be determined later.

Then $A(t)$ satisfies the inequality $A(t)^{2} \ll(b / c) A(t)$.

Now it is clear that $(2.10)_{1}$ is satisfied, if we choose $b$ sufficiently large.

Suppose that $(2.10)_{\mu-1}$ is satisfied for some $b$ and $c$. By the definition of $\phi_{\mu}(t)$

$$
\left\|\phi_{\mu}(t)\right\|_{(m)}^{\prime \prime} \ll\left\|\left(I-D_{0} L_{0}^{-1} \bar{\partial}_{F}^{(1) *} N_{F} L\right)\left(I-\rho H_{F}^{2} L\right) D_{1}^{*} N R_{2}\left(\phi^{\mu-1}(t)\right)\right\|_{(m)}^{\prime \prime} .
$$


By Proposition 1.2,

$$
\left\|\phi_{\mu}(t)\right\|_{(m)}^{\prime \prime} \ll c_{1}\left\|\phi^{\mu-1}(t)\right\|_{(m)}^{\prime 2}
$$

where $c_{1}$ is a constant independent of $\mu$.

By our assumption $(2.10)_{\mu-1}$, we have

$$
\left\|\phi_{\mu}(t)\right\|_{(m)}^{\prime \prime} \ll c_{1} A(t)^{2} \ll\left(b c_{1} / c\right) A(t) .
$$

Hence, if we choose $b$ and $c$ in such a way that $\left(b c_{1} / c\right) \leqq 1$ and $(2.10)_{1}$ are satisfied, we see that

$$
\left\|\phi^{\mu}(t)\right\|_{(m)}^{\prime \prime} \ll A(t) \quad \text { for all } \quad \mu \geqq 1,
$$

by induction on $\mu$.

Thus $\phi(t)=\lim _{\mu \rightarrow \infty} \phi^{\mu}(t)$ is convergent for $t$ with $|t|<\varepsilon$, for some $\varepsilon>0$, and it determines an element of $\Gamma_{(m)}^{\prime \prime}\left(M,{ }^{o} \bar{T}^{\prime \prime} \otimes\left({ }^{o} T^{\prime \prime}\right)^{*}\right)\left\{t_{1}, \ldots, t_{q}\right\}$. By Sobolev's lemma, $\phi(t)$ is of class $C^{2}$ because $m \geqq n+2$.

Then if we set $h(t)=\boldsymbol{H}_{T^{\prime}}^{2}(P(\phi(t))), h(t)$ is in $H_{T^{\prime}}^{(2)}\left\{t_{1}, \ldots, t_{q}\right\}$ and it satisfies

$$
\begin{aligned}
h(t) & \equiv \boldsymbol{H}_{T^{\prime}}^{2}\left(P\left(\phi^{\mu-1}(t)\right)\right) \quad \bmod \left(t^{\mu+1}\right) \\
& \equiv h^{\mu}(t) \quad \bmod \left(t^{\mu+1}\right) .
\end{aligned}
$$

Hence $\lim _{\mu \rightarrow \infty} h^{\mu}(t)$ coincides with $h(t)$.

From $(2.1 .1)_{\mu}$ and $(2.1 .2)_{\mu}$, we have

$$
\begin{aligned}
& D_{1} \phi(t)+R_{2}(\phi(t)) \equiv 0 \quad \bmod \left(h(t), t^{\mu+1}\right), \\
& L \phi(t) \equiv 0 \quad \bmod \left(h(t), t^{\mu+1}\right),
\end{aligned}
$$

for any $\mu \geqq 1$.

Let $T$ be the analytic subset of a smaller neighbourhood $V$ of 0 in $D=\{t \mid$ $|t|<\varepsilon\}$ defined by $h(t)=0$. Let $\tau: \widetilde{T} \rightarrow T$ be a resolution of the singularity of $T$ and $\left(t_{1}^{\prime}, \ldots, t_{q^{\prime}}^{\prime}\right)$ a system of local coordinates of $\widetilde{T}$ at $0^{\prime}$ for any $0^{\prime} \in \tau^{-1}(0)$.

From $(2.1 .1)_{\mu}^{\prime}$ and $(2.1 .2)_{\mu}^{\prime}$, we have, for any $\mu \geqq 0$,

$$
\begin{aligned}
& D_{1} \phi\left(\tau\left(t^{\prime}\right)\right)+R_{2}\left(\phi\left(\tau\left(t^{\prime}\right)\right)\right) \equiv 0 \quad \bmod \left(t^{\prime \mu+1}\right), \\
& L \phi\left(\tau\left(t^{\prime}\right)\right) \equiv 0 \bmod \left(t^{\prime \mu+1}\right) .
\end{aligned}
$$

Since the left hand sides of these equations are holomorphic in $t^{\prime}$, we infer that they all vanish. Hence (2.1.1) and (2.1.2) follow if we replace $T$ by a smaller neighbourhood of 0 in $T$. 


\section{§3. Proof of Versality}

The purpose of this section is to prove that the complex analytic family of $C^{2}$-partially complex structures on $M$ constructed in Section 2 is the versal family.

Let $(\mathscr{N}, \pi, S)$ be an arbitrary family of deformations of a neighbourhood $\tilde{N}$ of $M$ such that $\mathscr{N}_{o}=\tilde{N}$ for $o \in S$.

We may assume the following:

(3.i) $o$ is the origin of $\boldsymbol{C}^{r}$ and $S$ is an analytic subspace of a neighbourhood $D$ of $o$ in $\mathbb{C}^{r}$ defined by $b_{1}(s)=\cdots=b_{l}(s)=0$.

(3.ii) We find a finite system of open sets of $\mathscr{N},\left\{\mathscr{U}_{j}\right\}_{j \in \Lambda}$, such that there exists an analytic embedding

$$
\eta_{j}: \mathscr{U}_{j} \rightarrow W_{j} \times D \text { with } p_{2} \circ \eta_{j}=\pi \text { for each } j \in \Lambda,
$$

and $M \subset \cup_{j \in A} \mathscr{U}_{j}$, where $W_{j}$ is a neighbourhood of 0 in $\mathbb{C}^{n}$ and $p_{2}$ denotes the projection of $W_{j} \times D$ onto the second factor. We denote by $\zeta_{j}=\left(\zeta_{j}, \ldots, \zeta_{j}^{n}\right)$ and $s=\left(s_{1}, \ldots, s_{r}\right)$ the coordinates of $W_{j}$ and $D$ respectively, and set $z_{j}^{\lambda}=\zeta_{j}^{\lambda} \circ \eta_{j \mid \mathcal{N}_{0}}$ for $\lambda=1, \ldots, n$ and $U_{j}=\mathscr{U}_{j} \cap M$ where we regard $\zeta_{j}^{\lambda}$ as a function on $W_{j} \times D$.

(3.iii) $\eta_{j} \circ \eta_{k}^{-1}$ is represented by

$$
\begin{cases}\zeta_{j}^{\lambda}=f_{j k}^{\lambda}\left(\zeta_{k}, s\right) & \text { for } \quad \lambda=1, \ldots, n, \\ s_{\alpha}=s_{\alpha} & \text { for } \quad \alpha=1, \ldots, r,\end{cases}
$$

and we set $\bar{f}_{j k}^{\lambda}\left(z_{k}\right)=f_{j k}^{\lambda}\left(z_{k}, 0\right)$ for $\lambda=1, \ldots, n$.

From (3.iii), we infer that

(3.iv) $f_{i j}^{\lambda}\left(f_{j k}\left(\zeta_{k}, s\right), s\right) \equiv f_{i k}^{\lambda}\left(\zeta_{k}, s\right) \bmod (b(s))$ for $\lambda=1, \ldots, n$.

To prove the versality of the family constructed in Section 2, it suffices to show the existence of a neighbourhood $D^{\prime}$ of 0 in $D$, of a $\mathbb{C}^{2}$-family $g_{i}(s)$ of sections of $T^{\prime}$ over $U_{i}$ which depends complex analytically on $s$ in $D^{\prime}$ for each $i \in \Lambda$, and of a $\mathscr{H}$-valued holomorphic function $\tau(s)$ on $D^{\prime}$ satisfying;

$$
\begin{aligned}
& \left(g_{i}(0)\right)^{\lambda}=z_{i}^{\lambda} \quad \text { for } \lambda=1, \ldots, n \text { and } \tau(0)=0, \\
& \left(g_{i}(s)\right)^{\lambda}-f_{i j}^{\lambda}\left(g_{j}(s), s\right)=0 \quad \text { for } \quad s \in S^{\prime} \text { and } \lambda=1, \ldots, n, \\
& \left(\bar{\partial}_{b}+\phi(\tau(s))\right)\left(g_{i}(s)\right)^{\lambda}=0 \quad \text { for } \quad s \in S^{\prime} \text { and } \lambda=1, \ldots, n, \\
& h(\tau(s))=0 \quad \text { for } \quad s \in S^{\prime},
\end{aligned}
$$

where $S^{\prime}=D^{\prime} \cap S, g_{i}(s)$ has the expression $g_{i}(s)=\sum_{\lambda=1}^{n}\left(g_{i}(s)\right)^{\lambda} \partial / \partial z_{i}^{\lambda}$ regarded as an element of $\Gamma\left(U_{i}, T^{\prime} N_{\mid M}\right)$ since $T^{\prime}$ is isomorphic to $T^{\prime} N_{\mid M}$ as a $C^{\infty}$-vector 
bundle, and $\left(\bar{\partial}_{b}+\phi(\tau(s))\right)\left(g_{i}(s)\right)^{\lambda}$ denotes the element of $\Gamma\left(U_{i},\left({ }^{o} T^{\prime \prime}\right)^{*}\right)$ defined by the congruence

$$
\left(\bar{\partial}_{b}+\phi(\tau(s))\right)\left(g_{i}(s)\right)^{\lambda}(X)=\bar{\partial}_{b}\left(g_{i}(s)\right)^{\lambda}(X)+(\phi(\tau(s))(X))\left(g_{i}(s)\right)^{\lambda}
$$

for $X \in \Gamma\left(U_{i},\left({ }^{o} T^{\prime \prime}\right)\right)$.

\section{(I) Construction of a Formal Solution}

In this paragraph, we shall construct $\left\{g_{i}(s)\right\}_{i \in \Lambda}$ and $\tau(s)$ formally in $s$, that is to say, we construct sequences $\left\{g_{i}^{\mu}(s)\right\}_{i \in \Lambda}$ and $\tau^{\mu}(s)$ for $\mu=0,1, \ldots$, satisfying the following:

$$
\left(g_{i}^{0}\right)^{\lambda}=z_{i}^{\lambda} \quad \text { for } \quad \lambda=1, \ldots, n \text { and } \tau^{0}=0,
$$

for any $\mu \geqq 0$,

$(3.1)_{\mu} \quad\left(g_{i}^{\mu}(s)\right)^{\lambda}-f_{i j}^{\lambda}\left(g_{j}^{\mu}(s), s\right) \equiv 0 \quad \bmod \left(b(s), s^{\mu+1}\right) \quad$ for $\quad \lambda=1, \ldots, n$,

$(3.2)_{\mu} \quad\left(\check{\partial}_{b}+\phi\left(\tau^{\mu}(s)\right)\right)\left(g_{i}^{\mu}(s)\right)^{\lambda} \equiv 0 \bmod \left(b(s), s^{\mu+1}\right) \quad$ for $\quad \lambda=1, \ldots, n$,

$(3.3)_{\mu} \quad h\left(\tau^{\mu}(s)\right) \equiv 0 \quad \bmod \left(b(s), s^{\mu+1}\right)$,

$(3.4)_{\mu} g_{i}^{\mu}(s)$ is a $\Gamma\left(U_{i}, T^{\prime}\right)$-valued polynomial in $s$ of degree $\mu$ and $\tau^{\mu}(s)$ is

a $\mathscr{H}$-valued polynomial in $s$ of the same degree such that

$$
g_{i}^{\mu}(s) \equiv g_{i}^{\mu-1}(s) \bmod \left(s^{\mu}\right),
$$

and

$$
\tau^{\mu}(s) \equiv \tau^{\mu-1}(s) \bmod \left(s^{\mu}\right)
$$

We shall construct these $\left\{g_{i}^{\mu}(s)\right\}_{i \in A}$ and $\tau^{\mu}(s)$ by induction on $\mu$.

For $\mu=0$, because of (3.0), we set

$$
\left(g_{i}^{0}\right)^{\lambda}=z_{i}^{\lambda} \quad \text { for } \lambda=1, \ldots, n, \quad \text { and } \quad \tau^{0}=0 .
$$

Suppose that $\left\{g_{i}^{\mu-1}(s)\right\}_{i \in \Lambda}$ and $\tau^{\mu-1}(s)$ are determined for some $\mu \geqq 1$. First we define a $\Gamma\left(U_{i} \cap U_{j}, T^{\prime}\right)$-valued polynomial in $s$ of degree $\mu, \sigma_{i j}^{\mu}(s)$, by

$$
\sigma_{i j}^{\mu}(s) \equiv \sum_{\lambda=1}^{n}\left\{\left(g_{i}^{\mu-1}(s)\right)^{\lambda}-f_{i j}^{\lambda}\left(g_{j}^{\mu-1}(s), s\right)\right\} \partial / \partial z_{i}^{\lambda} \bmod \left(s^{\mu+1}\right) .
$$

Then we set

$$
g_{i \mid \mu}^{\prime}(s)=\sum_{k \in A} \rho_{k} \kappa_{s}^{\mu}\left(\sigma_{k i}^{\mu}(s)\right),
$$

where $\left\{\rho_{k}\right\}_{k \in \Lambda}$ is a partition of unity subordinate to $\left\{U_{k}\right\}_{k \in \Lambda}$. Next we define $\Gamma\left(U_{i}, T^{\prime} \otimes\left({ }^{\circ} T^{\prime \prime}\right)^{*}\right)$-valued polynomials $\omega_{i}^{\mu}(s)$ and $\xi_{i}^{\mu}(s)$ of degree $\mu$ by

$$
\omega_{i}^{\mu}(s) \equiv-\sum_{\lambda=1}^{n}\left[\bar{\partial}_{b}\left\{\left(g_{i}^{\mu-1}(s)\right)^{\lambda}+\left(g_{i \mid \mu}^{\prime}(s)\right)^{\lambda}\right\}\right.
$$




$$
\left.+\phi\left(\tau^{\mu-1}(s)\right)\left\{\left(g_{i}^{\mu-1}(s)\right)^{\lambda}-\left(g_{i}^{0}\right)^{\lambda}\right\}\right] \partial / \partial z_{i}^{\lambda} \bmod \left(s^{\mu+1}\right)
$$

and

$$
\xi_{i}^{\mu}(s) \equiv \omega_{i}^{\mu}(s)-\phi\left(\tau^{\mu-1}(s)\right)_{\mid U_{i}} \bmod \left(s^{\mu+1}\right)
$$

Then we set

$$
\tau_{\mu}(s)=\sum_{\sigma=1}^{q} \tau_{\mu}^{\sigma}(s)\left(\mathscr{L} \beta_{\sigma}\right)=\mathscr{L} \mathbf{H}_{T^{\prime}}^{1}\left(\sum_{i \in \Lambda} \rho_{i} \kappa_{s}^{\mu}\left(\xi_{i}^{\mu}(s)\right)\right)
$$

and

$$
g_{\mu}^{\prime}(s)=-\bar{\partial}_{T^{\prime}}^{(0) * *} N_{T^{\prime}}\left(\sum_{i \in \Lambda} \rho_{i} \kappa_{s}^{\mu}\left(\xi_{i}^{\mu}(s)\right)-\tau_{\mu}(s)\right) .
$$

Then we infer that $g_{\mu}^{\prime}(s)$ is $\Gamma\left(M, T^{\prime}\right)$-valued, since $N_{T^{\prime}}$ is a $C^{\infty}$-operator. Finally we set

$$
g_{i}^{\mu}(s)=g_{i}^{\mu-1}(s)+g_{i \mid \mu}^{\prime}(s)+g_{\mu}^{\prime}(s)
$$

and

$$
\tau^{\mu}(s)=\tau^{\mu-1}(s)+\tau_{\mu}(s) .
$$

It is clear that (3.0) and (3.4) are satisfied for all $\mu \geqq 1$.

Proposition 3.1. For any $\mu \geqq 0$,

(1) ${ }_{\mu}\left(g_{i}^{\mu}(s)\right)^{\lambda}-f_{i j}^{\lambda}\left(g_{j}^{\mu}(s), s\right) \equiv 0 \quad \bmod \left(b(s), s^{\mu+1}\right) \quad$ for $\quad \lambda=1, \ldots, n$,

(2) $\theta_{i}^{\mu}(s)-\phi\left(\tau^{\mu}(s)\right)_{\mid U_{i}} \equiv 0 \bmod \left(b(s), s^{\mu+1}\right)$

where $\theta_{i}^{\mu}(s)$ is a $\Gamma\left(U_{i}, T^{\prime} \otimes\left({ }^{o} T^{\prime \prime}\right)^{*}\right)$-valued polynomial in $s$ of degree $\mu$ defined by

$$
\left(\bar{\partial}_{b}+\theta_{i}^{\mu}(s)\right)\left(g_{i}^{\mu}(s)\right)^{\lambda} \equiv 0 \quad \bmod \left(s^{\mu+1}\right) \quad \text { for } \quad \lambda=1, \ldots, n,
$$

and $\left(\bar{\partial}_{b}+\theta_{i}^{\mu}(s)\right)\left(g_{i}^{\mu}(s)\right)^{\lambda}$ is defined by the same congruence of $\left(\bar{\partial}_{b}+\phi(\tau(s))\right)$ $\times\left(g_{i}(s)\right)^{\lambda}$.

(3) $\quad h\left(\tau^{\mu}(s)\right) \equiv 0 \quad \bmod \left(b(s), s^{\mu+1}\right)$,

(4) $\sum_{\mu \in \Lambda} \rho_{k}\left\{\sigma_{k j}^{\mu+1}(s)-\sigma_{k i}^{\mu+1}(s)\right\} \equiv 0 \quad \bmod \left(s^{\mu+1}\right)$,

(5) ${ }_{\mu} \quad \bar{\partial}_{T^{\prime}}^{(0)} \bar{\partial}_{T^{\prime}}^{(0) *} N_{T^{\prime}}\left\{\sum_{i \in \Lambda} \rho_{i} \omega_{i}^{\mu+1}-\phi\left(\tau^{\mu}(s)\right)\right\} \equiv 0 \bmod \left(s^{\mu+1}\right)$,

(6) $\boldsymbol{H}_{T}^{1},\left\{\sum_{i \in \Lambda} \rho_{i} \omega_{i}^{\mu+1}(s)-\phi\left(\tau^{\mu}(s)\right)\right\} \equiv 0 \bmod \left(s^{\mu+1}\right)$.

Proof. For $\mu=0$, it is clear that $(1)_{0}-(3)_{0}$ are satisfied. Since

$$
\begin{array}{ll}
\sigma_{i j}^{1}(s) \equiv 0 & \bmod (s), \\
\omega_{i}^{1}(s) \equiv 0 & \bmod (s),
\end{array}
$$

and 


$$
\phi\left(\tau^{0}(s)\right)=0
$$

$(4)_{0}-(6)_{0}$ are also satisfied.

We suppose that $(1)_{\mu-1}-(6)_{\mu-1}$ are satisfied for some $\mu \geqq 1$. To prove $(1)_{\mu}$, we show the following lemma.

Lemma 3.2. $\sigma_{k i}^{\mu}(s)-\sigma_{k j}^{\mu}(s)+\sigma_{i j}^{\mu}(s) \equiv 0 \quad \bmod \left(b(s), s^{\mu+1}\right)$.

Proof. Omitting the index subordinate to the local base $\left(\partial / \partial z_{i}^{1}, \ldots, \partial / \partial z_{i}^{n}\right)$ of $T^{\prime}$,

$$
\begin{aligned}
\sigma_{k i}^{\mu}(s) \equiv & g_{k}^{\mu-1}(s)-f_{k i}\left(g_{i}^{\mu-1}(s), s\right) \bmod \left(s^{\mu+1}\right) \\
\equiv & g_{k}^{\mu-1}(s)-f_{k i}\left(f_{i j}\left(g_{j}^{\mu-1}(s), s\right), s\right) \\
& -\sum_{\lambda=1}^{n}\left(\partial f_{k i} / \partial \zeta_{i}^{\lambda}\right)\left(f_{i j}\left(g_{j}^{\mu-1}(s), s\right), s\right)\left(\sigma_{i j}^{\mu}(s)\right)^{\lambda} \bmod \left(s^{\mu+1},\left(\sigma_{i j}^{\mu}(s)\right)^{2}\right) \\
\equiv & \sigma_{k j}^{\mu}(s)-\sum_{\lambda=1}^{n}\left(\partial \bar{f}_{k i} / \partial z_{i}^{\lambda}\right)\left(z_{i}\right)\left(\sigma_{i j}^{\mu}(s)\right)^{\lambda} \bmod \left(s^{\mu+1},\left(\sigma_{i j}^{\mu}(s)\right)^{2}, s \sigma_{i j}^{\mu}(s), b(s)\right) .
\end{aligned}
$$

From $(1)_{\mu-1}, \sigma_{i j}^{\mu}(s) \equiv 0 \bmod \left(b(s), s^{\mu}\right)$. Then Lemma 3.2 follows.

$$
\begin{aligned}
& \text { Proof of }(1)_{\mu}: g_{i}^{\mu}(s)-f_{i j}\left(g_{j}^{\mu}(s), s\right) \\
& \equiv g_{i}^{\mu-1}(s)+g_{i \mid \mu}^{\prime}(s)+g_{\mu}^{\prime}(s)-f_{i j}\left(g_{j}^{\mu-1}(s), s\right) \\
& \quad-\sum_{\lambda=1}^{n}\left(\partial \bar{f}_{i j} / \partial z_{j}^{\lambda}\right)\left(z_{j}\right)\left\{\left(g_{j \mid \mu}^{\prime}(s)\right)^{\lambda}+\left(g_{\mu}^{\prime}(s)\right)^{\lambda}\right\} \quad \bmod \left(s^{\mu+1}\right) \\
& \equiv \sigma_{i j}^{\mu}(s)-\sum_{k \in \Lambda} \rho_{k} \kappa_{s}^{\mu}\left(\sigma_{k j}^{\mu}(s)-\sigma_{k i}^{\mu}(s)\right) \bmod \left(s^{\mu+1}\right), \\
& \left.\quad \quad \quad \text { by the definition of } \mathbf{g}_{i \mid \mu}^{\prime}(s) \text { and } g_{\mu}^{\prime}(s)\right), \\
& \equiv \sigma_{i j}^{\mu}(s)-\sum_{k \in \Lambda} \rho_{k}\left(\sigma_{k j}^{\mu}(s)-\sigma_{k i}^{\mu}(s)\right) \bmod \left(s^{\mu+1}\right), \quad\left(\text { by }(4)_{\mu-1}\right), \\
& \equiv 0 \quad \bmod \left(b(s), s^{\mu+1}\right), \quad(\text { by Lemma } 3.2) .
\end{aligned}
$$

Proof of $(4)_{\mu}$ : As we have proved above,

$$
\sigma_{k j}^{\mu+1}(s) \equiv \sigma_{k j}^{\mu}(s)-\sum_{l \in \Lambda} \rho_{l}\left(\sigma_{l j}^{\mu}(s)-\sigma_{l k}^{\mu}(s)\right) \bmod \left(s^{\mu+1}\right) .
$$

Hence

$$
\sigma_{k j}^{\mu+1}(s)-\sigma_{k i}^{\mu+1}(s) \equiv \sigma_{k j}^{\mu}(s)-\sigma_{k i}^{\mu}(s)-\sum_{l \in \Lambda} \rho_{l}\left(\sigma_{l j}^{\mu}(s)-\sigma_{l i}^{\mu}(s)\right) \bmod \left(s^{\mu+1}\right)
$$

Then,

$$
\sum_{k \in \Lambda} \rho_{k}\left(\sigma_{k j}^{\mu+1}(s)-\sigma_{k i}^{\mu+1}(s)\right) \equiv 0 \quad \bmod \left(s^{\mu+1}\right)
$$

To prove $(2)_{\mu},(3)_{\mu}$, we prove some lemmas.

Lemma 3.3. $\theta_{i}^{\mu}(s) \equiv \omega_{i}^{\mu}(s)-\bar{\partial}_{T^{\prime}}^{(0)} g_{\mu}^{\prime}(s)_{\mid U_{i}} \bmod \left(b(s), s^{\mu+1}\right)$.

Proof. From the definition of $\theta_{i}^{\mu}(s)$ and $\omega_{i}^{\mu}(s)$,

$$
\begin{aligned}
\bar{\partial}_{b}\left\{\left(g_{i}^{\mu-1}(s)\right)^{\lambda}\right. & \left.+\left(g_{i \mid \mu}^{\prime}(s)\right)^{\lambda}\right\}+\bar{\partial}_{b}\left(g_{\mu}^{\prime}(s)\right)^{\lambda}{ }_{\mid U_{i}}+\left(\theta_{i}^{\mu}(s)\right)^{\lambda} \\
& +\theta_{i}^{\mu}(s)\left\{\left(g_{i}^{\mu-1}(s)\right)^{\lambda}-\left(g_{i}^{0}\right)^{\lambda}\right\} \equiv 0 \bmod \left(s^{\mu+1}\right)
\end{aligned}
$$


and

$$
\begin{aligned}
\bar{\partial}_{b}\left\{\left(g_{i}^{\mu-1}(s)\right)^{\lambda}\right. & \left.+\left(g_{i \mid \mu}^{\prime}(s)\right)^{\lambda}\right\}+\left(\omega_{i}^{\mu}(s)\right)^{\lambda} \\
& +\phi\left(\tau^{\mu-1}(s)\right)\left\{\left(g_{i}^{\mu-1}(s)\right)^{\lambda}-\left(g_{i}^{0}\right)^{\lambda}\right\} \equiv 0 \bmod \left(s^{\mu+1}\right)
\end{aligned}
$$

for $\lambda=1, \ldots, n$. From $(2)_{\mu-1}$, we infer that

$$
\left(\theta_{i}^{\mu}(s)\right)^{\lambda} \equiv\left(\omega_{i}^{\mu}(s)\right)^{\lambda}-\bar{c}_{b}\left(g_{\mu}^{\prime}(s)_{\mid U_{i}}\right)^{\lambda} \quad \bmod \left(b(s), s^{\mu+1}\right) \quad \text { for } \quad \lambda=1, \ldots, n .
$$

This yields Lemma 3.3.

Corollary 3.4. $P\left(\omega_{i}^{\mu}(s)\right) \equiv 0 \quad \bmod \left(b(s), s^{\mu+1}\right)$.

Proof. Let $\theta_{i}(s)$ be a complex analytic family of elements of $\Gamma\left(U_{i}\right.$, $\left.T^{\prime} \otimes\left({ }^{\circ} T^{\prime \prime}\right)^{*}\right)$ parametrized by $s$ in a sufficiently small neighbourhood of 0 in $D$, defined by

$$
\left(\bar{\partial}_{b}+\theta_{i}(s)\right)\left(g_{i}^{\mu}(s)\right)^{\lambda}=0 \quad \text { for } \quad \lambda=1, \ldots, n,
$$

where $\left(\bar{\partial}_{b}+\theta_{i}(s)\right)\left(g_{i}^{\mu}(s)\right)^{\lambda}$ is defined by the same congruence of $\left(\bar{\partial}_{b}+\phi(\tau(s))\right)$ $\times\left(g_{i}(s)\right)^{\lambda}$.

Since, for each $s, \theta_{i}(s)$ represents the partially complex structure on $U_{i}$ induced from the complex structure on $\mathbb{C}^{n}$ via a $C^{\infty}$-embedding $g_{i}^{\mu}(s)$, it is clear that

$$
P\left(\theta_{i}(s)\right)=0 .
$$

Since $\theta_{i}^{\mu}(s) \equiv \theta_{i}(s) \bmod \left(s^{\mu+1}\right)$, (by the definition of $\left.\theta_{i}^{\mu}(s)\right)$, and $\omega_{i}^{\mu}(s) \equiv \theta_{i}^{\mu}(s)$ $+\bar{\partial}_{T^{\prime}}^{(0)} g_{\mu}^{\prime}(s)_{\mid U_{i}} \bmod \left(b(s), s^{\mu+1}\right)$, (by Lemma 3.3$)$,

$$
\begin{aligned}
P\left(\omega_{i}^{\mu}(s)\right) & \equiv P\left(\theta_{i}^{\mu}(s)+\bar{\partial}_{T^{\prime}}^{(0)} g_{\mu}^{\prime}(s)_{\mid U_{i}}\right) \quad \bmod \left(b(s), s^{\mu+1}\right) \\
& \equiv P\left(\theta_{i}^{\mu}(s)\right) \quad \bmod \left(s^{\mu+1}\right) \\
& \equiv P\left(\theta_{i}(s)\right) \quad \bmod \left(s^{\mu+1}\right) \\
& =0
\end{aligned}
$$

Lemma 3.5. $\theta_{i}^{\mu}(s) \equiv \theta_{j}^{\mu}(s) \quad \bmod \left(b(s), s^{\mu+1}\right) \quad$ on $\quad U_{i} \cap U_{j}$.

Proof. $\left(\bar{\partial}_{b}+\theta_{j}^{\mu}(s)\right)\left(g_{i}^{\mu}(s)\right)$

$$
\begin{aligned}
& \equiv \sum_{\lambda=1}^{n}\left(\partial f_{i j} / \partial \zeta_{j}^{\lambda}\right)\left(g_{j}^{\mu}(s), s\right)\left(\bar{\partial}_{b}+\theta_{j}^{\mu}(s)\right)\left(g_{j}^{\mu}(s)\right)^{\lambda} \bmod \left(b(s), s^{\mu+1}\right), \\
& \equiv 0 \bmod \left(b(s), s^{\mu+1}\right), \quad\left(\text { from the definition of } \theta_{j}^{\mu}(s)\right) .
\end{aligned}
$$

Since $\theta_{i}^{\mu}(s)$ is determined uniquely by

$$
\left(\bar{\partial}_{b}+\theta_{i}^{\mu}(s)\right)\left(g_{i}^{\mu}(s)\right)^{\lambda} \equiv 0 \bmod \left(s^{\mu+1}\right) \quad \text { for } \quad \lambda=1, \ldots, n,
$$


Lemma 3.5 follows.

From Lemma 3.3 and Lemma 3.5, we have the following corollary.

Corollary 3.6. $\omega_{i}^{\mu}(s) \equiv \omega_{j}^{\mu}(s) \quad \bmod \left(b(s), s^{\mu+1}\right) \quad$ on $\quad U_{i} \cap U_{j}$.

Lemma 3.7. $h\left(\tau^{\mu-1}(s)\right) \equiv 0 \bmod \left(b(s), s^{\mu+1}\right)$.

Proof. By the definition of $h(t)$ in Section 2 (II), we have

$$
h\left(\tau^{\mu-1}(s)\right)=\boldsymbol{H}_{T}^{2}, P\left(\phi\left(\tau^{\mu-1}(s)\right)\right) .
$$

Since

$$
\begin{aligned}
& P\left(\phi\left(\tau^{\mu-1}(s)\right)\right)=\sum_{i \in \Lambda} \rho_{i} P\left(\phi\left(\tau^{\mu-1}(s)\right)_{\mid U_{i}}\right) \\
& \equiv \sum_{i \in \Lambda} \rho_{i} P\left(\omega_{i}^{\mu}(s)-\xi_{i}^{\mu}(s)\right) \bmod \left(s^{\mu+1}\right), \\
& P\left(\omega_{i}^{\mu}(s)-\xi_{i}^{\mu}(s)\right) \equiv P\left(\omega_{i}^{\mu}(s)\right)-\bar{\partial}_{T^{\prime}}^{(1)} \xi_{i}^{\mu}(s) \bmod \left(b(s), s^{\mu+1}\right),
\end{aligned}
$$

(by Lemma 3.3 and $(2)_{\mu-1}$ ), and

$$
\begin{aligned}
& P\left(\omega_{i}^{\mu}(s)\right) \equiv 0 \quad \bmod \left(b(s), s^{\mu+1}\right), \quad(\text { by Corollary } 3.4) \\
& h\left(\tau^{\mu-1}(s)\right) \equiv-\boldsymbol{H}_{T^{\prime}}^{2}\left\{\bar{\partial}_{T^{\prime}}^{(1)}\left(\sum_{i \in \Lambda} \rho_{i} \xi_{i}^{\mu}(s)\right)\right\} \quad \bmod \left(b(s), s^{\mu+1}\right) \\
& =0
\end{aligned}
$$

Lemma 3.8. $\quad \bar{\partial}_{T^{\prime}}^{(1)}\left(\sum_{i \in \Lambda} \rho_{i} \breve{\zeta}_{i}^{\mu}(s)\right) \equiv 0 \quad \bmod \left(b(s), s^{\mu+1}\right)$.

Proof. By the definition of $\xi_{i}^{\mu}(s)$, we have

$$
\bar{\partial}_{T^{\prime}}^{(1)} \xi_{i}^{\mu}(s) \equiv \bar{\partial}_{T^{\prime}}^{(1)} \omega_{i}^{\mu}(s)-\bar{\partial}_{T^{\prime}}^{(1)} \phi\left(\tau^{\mu-1}(s)\right)_{\mid U_{i}} \bmod \left(s^{\mu+1}\right) .
$$

Since

$$
P\left(\omega_{i}^{\mu}(s)\right) \equiv 0 \quad \bmod \left(b(s), s^{\mu+1}\right)
$$

and

$$
\begin{aligned}
& P\left(\phi\left(\tau^{\mu-1}(s)\right)\right) \equiv 0 \quad \bmod \left(h\left(\tau^{\mu-1}(s)\right), s^{\mu+1}\right) \\
& \bar{\partial}_{T^{\prime}}^{(1)} \xi_{i}^{\mu}(s) \equiv P\left(\omega_{i}^{\mu}(s)\right)-P\left(\omega_{i}^{\mu}(s)-\xi_{i}^{\mu}(s)\right) \bmod \left(b(s), s^{\mu+1}\right) \\
& \equiv P\left(\omega_{i}^{\mu}(s)\right)-P\left(\phi\left(\tau^{\mu-1}(s)\right)\right)_{\mid U_{\imath}} \bmod \left(b(s), s^{\mu+1}\right), \quad\left(\text { by the definition of } \xi_{i}^{\mu}(s)\right) \\
& \equiv 0 \bmod \left(b(s), s^{\mu+1}, h\left(\tau^{\mu-1}(s)\right)\right) \\
& \equiv 0 \quad \bmod \left(b(s), s^{\mu+1}\right), \quad(\text { by Lemma } 3.7)
\end{aligned}
$$

Hence, taking account of Corollary 3.6,

$$
\begin{aligned}
\bar{\partial}_{T^{\prime}}^{(1)}\left(\sum_{i \in \Lambda} \rho_{i} \xi_{i}^{\mu}(s)\right) & \equiv \sum_{i \in \Lambda} \rho_{i} \overline{\hat{\sigma}}_{T^{\prime}}^{(1)} \xi_{i}^{\mu}(s) \bmod \left(b(s), s^{\mu+1}\right) & \\
& \equiv 0 \bmod \left(b(s), s^{\mu+1}\right) . & \text { Q. E. D. }
\end{aligned}
$$

Lemma 3.9. $\sum_{i \in \Lambda} \rho_{i} \theta_{i}^{\mu}(s)-\phi\left(\tau^{\mu}(s)\right) \equiv 0 \bmod \left(b(s), s^{\mu+1}\right)$. 
Proof. By Lemma 3.3, we have

$$
\begin{aligned}
& \sum_{i \in A} \rho_{i} \theta_{i}^{\mu}(s)-\phi\left(\tau^{\mu}(s)\right) \\
& \equiv \sum_{i \in \Lambda} \rho_{i} \omega_{i}^{\mu}(s)+\bar{\partial}_{T^{\prime}}^{(0)} g_{\mu}^{\prime}(s)-\phi\left(\tau^{\mu-1}(s)\right)-\phi_{1} \tau_{\mu}(s) \bmod \left(b(s), s^{\mu+1}\right) \\
& \equiv \sum_{i \in \Lambda} \rho_{i} \xi_{i}^{\mu}(s)-\overline{\hat{o}}_{T^{\prime}}^{(0)} \overline{\widehat{c}}_{T^{\prime}}^{(0) *} N_{T^{\prime}}\left\{\sum_{i \in \Lambda} \rho_{i} \kappa_{s}^{\mu}\left(\xi_{i}^{\mu}(s)\right)\right. \\
& \left.-\mathscr{L} \boldsymbol{H}_{T^{\prime}}^{1}\left(\sum_{i \in \Lambda} \rho_{i} \kappa_{s}^{\mu}\left(\xi_{i}^{\mu}(s)\right)\right)\right\}-\mathscr{L} \boldsymbol{H}_{1}^{1},\left(\sum_{i \in \Lambda} \rho_{i} \kappa_{s}^{\mu}\left(\xi_{i}^{\mu}(s)\right)\right) \\
& \bmod \left(b(s), s^{\mu+1}\right) \text {, (from the definition of } g_{\mu}^{\prime}(s) \text { and } \tau_{\mu}(s) \text { ), } \\
& =\sum_{i \in \Lambda} \rho_{i} \xi_{i}^{\mu}(s)-\bar{\partial}_{T^{\prime}}^{(0)} \bar{\partial}_{T^{\prime}}^{(0) *} N_{T^{\prime}}\left(\sum_{i \in \Lambda} \rho_{i} \kappa_{s}^{\mu}\left(\xi_{i}^{\mu}(s)\right)\right) \\
& \left.-\bar{\partial}_{T}^{(0)} \bar{\partial}_{1}^{(0) *} N_{T^{\prime}} \bar{\partial}_{T^{\prime}}^{(0)} \theta_{\boldsymbol{H}_{T^{\prime}}^{\prime}\left(\Sigma_{i} \in A\right.} \rho_{i} \kappa_{s}^{\mu}\left(\xi_{i}^{\mu}(s)\right)\right) \\
& \left.-\boldsymbol{H}_{T}^{1},\left(\sum_{i \in \Lambda} \rho_{i} \kappa_{s}^{\mu}\left(\xi_{i}^{\mu}(s)\right)\right)+\bar{\partial}_{T}^{(0)} \theta_{H_{T}^{1} \cdot\left(\sum_{i} \in \Lambda \rho_{i}\right.} \kappa_{s}^{\mu}\left(\xi_{i}^{\mu}(s)\right)\right), \\
& \equiv \sum_{i \in \Lambda} \rho_{i} \xi_{i}^{\mu}(s)-\bar{\partial}_{T}^{(0)} \bar{c}_{T}^{(0)} * N_{T}\left(\sum_{i \in \Lambda} \rho_{i} \xi_{i}^{\mu}(s)\right)-\boldsymbol{H}_{T^{\prime}}^{1}\left(\sum_{i \in \Lambda} \rho_{i} \xi_{i}^{\mu}(s)\right) \\
& \bmod \left(s^{\mu+1}\right), \quad\left(\text { by }(5)_{\mu-1} \text { and }(6)_{\mu-1}\right) \text {, } \\
& =\bar{\partial}_{T^{\prime}}^{(1) *} \bar{\partial}_{T^{\prime}}^{(1)} N_{T^{\prime}}\left(\sum_{i \in \Lambda} \rho_{i} \xi_{i}^{\mu}(s)\right) \\
& \equiv 0 \bmod \left(b(s), s^{\mu+1}\right), \quad \text { (by Lemma 3.8). }
\end{aligned}
$$

Proof of $(2)_{\mu}$ : From Lemma 3.5, we infer that

$$
\left\{\sum_{j \in \Lambda} \rho_{j} \theta_{j}^{\mu}(s)\right\}_{\mid U_{i}} \equiv \theta_{i}^{\mu}(s) \bmod \left(b(s), s^{\mu+1}\right) .
$$

Then from Lemma 3.9, we have

$$
\theta_{i}^{\mu}(s) \equiv \phi\left(\tau^{\mu}(s)\right)_{\mid U_{i}} \bmod \left(b(s), s^{\mu+1}\right) .
$$

Proof of $(3)_{\mu}$ : Since the linear term of $h(t)$ is null, we have $h\left(\tau^{\mu}(s)\right)$ $\equiv h\left(\tau^{\mu-1}(s)\right) \bmod \left(s^{\mu+1}\right) \equiv 0 \bmod \left(b(s), s^{\mu+1}\right)$, (by Lemma 3.7).

Proof of $(5)_{\mu}: \quad \bar{\partial}_{T^{\prime}}^{(0)} \bar{\partial}_{T^{\prime}}^{(0) *} N_{T^{\prime}}\left\{\sum_{i \in A} \rho_{i} \omega_{i}^{\mu+1}(s)-\phi\left(\tau^{\mu}(s)\right)\right\}$ $\equiv \bar{\partial}_{T^{\prime}}^{(0)} \bar{\partial}_{T^{\prime}}^{(0) *} N_{T^{\prime}}\left\{\sum_{i \in \Lambda} \rho_{i} \omega_{i}^{\mu}(s)+\bar{\partial}_{T^{\prime}}^{(0)} g_{\mu}^{\prime}(s)-\phi\left(\tau^{\mu-1}(s)\right)-\phi_{1} \tau_{\mu}(s)\right\}$ $\bmod \left(s^{\mu+1}\right)$, (by the definition of $\omega_{i}^{\mu+1}(s)$ ), $\equiv \bar{\partial}_{T^{\prime}}^{(0)} \bar{\partial}_{T^{\prime}}^{(0) *} N_{T^{\prime}}\left(\sum_{i \in \Lambda} \rho_{i} \xi_{i}^{\mu}(s)\right)-\bar{\partial}_{T^{\prime}}^{(0)} \bar{\partial}_{T^{\prime}}^{(0) *} N_{T^{\prime}}\left\{\sum_{i \in \Lambda} \rho_{i} \kappa_{s}^{\mu}\left(\xi_{i}^{\mu}(s)\right)-\phi_{1} \tau_{\mu}(s)\right\}$ $-\bar{\partial}_{T^{\prime}}^{(0)} \bar{\partial}_{T^{\prime}}^{(0) *} N_{T^{\prime}} \phi_{1} \tau_{\mu}(s) \bmod \left(s^{\mu+1}\right), \quad$ (by the definition of $g_{\mu}^{\prime}(s)$ ), $\equiv 0 \bmod \left(s^{\mu+1}\right), \quad\left(\right.$ by $\left.(5)_{\mu-1}\right)$.

Proof of $(6)_{\mu}: \boldsymbol{H}_{T^{\prime}}^{1}\left\{\sum_{i \in \Lambda} \rho_{i} \omega_{i}^{\mu+1}(s)-\phi\left(\tau^{\mu}(s)\right)\right\}$

$$
\begin{aligned}
\equiv & \boldsymbol{H}_{T^{\prime}}^{1}\left\{\sum_{i \in \Lambda} \rho_{i} \xi_{i}^{\mu}(s)-\bar{\partial}_{T^{\prime}}^{(0)} \bar{\partial}_{T^{\prime}}^{(0) *} N_{T^{\prime}}\left(\sum_{i \in \Lambda} \rho_{i} \kappa_{s}^{\mu}\left(\xi_{i}^{\mu}(s)\right)-\phi_{1} \tau_{\mu}(s)\right)\right. \\
& \left.-\mathscr{L} \boldsymbol{H}_{T^{\prime}}^{1}\left(\sum_{i \in \Lambda} \rho_{i} \kappa_{s}^{\mu}\left(\xi_{i}^{\mu}(s)\right)\right)\right\} \bmod \left(s^{\mu+1}\right) \\
= & H_{T^{\prime}}^{1}\left(\sum_{i \in \Lambda} \rho_{i} \xi_{i}^{\mu}(s)\right)-\boldsymbol{H}_{T^{\prime}}^{1}\left(\sum_{i \in \Lambda} \rho_{i} \kappa_{s}^{\mu}\left(\xi_{i}^{\mu}(s)\right)\right), \quad\left(\text { since } \mathbb{H}_{T^{\prime}}^{1} \mathscr{L}=\mathbb{H}_{T^{\prime}}^{\prime}\right), \\
\equiv & 0 \bmod \left(s^{\mu+1}\right), \quad\left(\text { by }(6)_{\mu-1}\right) .
\end{aligned}
$$

By Proposition 3.1, we have (3.1) $)_{\mu}$ and $(3.3)_{\mu}$ for any $\mu \geqq 0$. From (2) $)_{\mu}$ in 
Proposition 3.1, we infer that, for any $\mu \geqq 0$,

$(3.2)_{\mu} \quad\left(\bar{\partial}_{b}+\phi\left(\tau^{\mu}(s)\right)\right)\left(g_{i}^{\mu}(s)\right)^{\lambda} \equiv 0 \bmod \left(b(s), s^{\mu+1}\right) \quad$ for $\quad \lambda=1, \ldots, n$.

This completes the inductive construction of $g_{i}^{\mu}(s)$ and $\tau^{\mu}(s)$.

\section{(II) Proof of Convergence}

In this paragraph, we prove that the formal power series $g_{i}(s)=\lim _{\mu \rightarrow \infty} g_{i}^{\mu}(s)$ and $\tau(s)=\lim _{\mu \rightarrow \infty} \tau^{\mu}(s)$ are convergent with respect to \|\|$_{(m)}^{\prime}$-norm and | |-norm respectively where $m \geqq n+2$ and || denotes the euclidean norm on the finite dimensional vector space $\mathscr{H}$.

To prove that $\left\{g_{i}^{\mu}(s)\right\}_{i \in \Lambda}$ and $\tau^{\mu}(s)$ are convergent, it suffices to show the following estimates: for all $\mu \geqq 1$,

$$
\begin{aligned}
& \left\|g_{i}^{\mu}(s)-g_{i}^{0}\right\|_{(m)}^{\prime} \ll A(s), \\
& \left|\tau^{\mu}(s)\right| \ll A(s),
\end{aligned}
$$

for some convergent power series $A(s)$.

Similarly to Section 2 (II), we set

$$
A(s)=(b / 16 c) \sum_{\mu=1}^{\infty}\left(c^{\mu} / \mu^{2}\right)\left(s_{1}+\cdots+s_{r}\right)^{\mu},
$$

and show that (3.5) $)_{\mu}$ and (3.6) $)_{\mu}$ are satisfied for suitable $b$ and $c$.

Since $\phi(t)$ is holomorphic in $t$ and $f_{i j}\left(\zeta_{j}, s\right)$ is holomorphic in $\left(\zeta_{j}, s\right)$, we may assume the following:

(3.v) $\|\phi(t)\|_{(m)} \ll\left(b_{0} / c_{0}\right) \sum_{\mu=1}^{\infty} c_{0}^{\mu}\left(t_{1}+\cdots+t_{q}\right)^{\mu}$,

(3.vi) $\| f_{i j}^{\lambda}\left(z_{j}+x, s\right)-\bar{f}_{i j}^{\lambda}\left(z_{j}\right)-\sum_{v=1}^{n}\left(\partial \bar{f}_{i j}^{\lambda} / \partial z_{j}^{v}\right)\left(z_{j}\right) x^{v}$

$$
-\sum_{\alpha=1}^{r}\left(\partial f_{i j}^{\lambda} / \partial s_{\alpha}\right)\left(z_{j}, 0\right) s_{\alpha} \|_{(m)}^{\prime}
$$

$$
\ll\left(b_{0} / c_{0}\right) \sum_{\mu=2}^{\infty} c_{0}^{\mu}\left(x^{1}+\cdots+x^{n}+s_{1}+\cdots+s_{r}\right)^{\mu} \quad \text { for } \quad \lambda=1, \ldots, n \text {. }
$$

For $\mu=1$, we can choose $b$ so large that (3.5) $)_{1}$ and (3.6) $)_{1}$ are satisfied.

We suppose that (3.5) $)_{\mu-1}$ and (3.6) $)_{\mu-1}$ are both satisfied for some $\mu \geqq 2$. Under this situation we claim the following lemma.

Lemma 3.10. For sufficiently large $c$, the following estimates are satisfied.

(1) $\left\|\kappa_{s}^{\mu}\left(\sigma_{i j}^{\mu}(s)\right)\right\|_{(m)}^{\prime} \ll(b / c) K_{1} A(s)$,

(2) $\left\|g_{i \mid \mu}^{\prime}(s)\right\|_{(m)}^{\prime} \ll(b / c) K_{2} A(s)$,

(3) $\left\|\kappa_{s}^{\mu}\left(\omega_{i}^{\mu}(s)\right)\right\|_{(m)} \ll(b / c) K_{3} A(s)$,

(4) $\left\|\kappa_{s}^{\mu}\left(\xi_{i}^{\mu}(s)\right)\right\|_{(m)} \ll(b / c) K_{4} A(s)$, 
where $K_{1}-K_{4}$ are constants independent of $\mu$.

Proof. (1) By the definition of $\sigma_{i j}^{\mu}(s)$,

$$
\begin{aligned}
\kappa_{s}^{\mu}\left(\sigma_{i j}^{\mu}(s)\right)= & \sum_{\lambda=1}^{n}-\kappa_{s}^{\mu}\left\{f_{i j}^{\lambda}\left(g_{j}^{\mu-1}(s), s\right)-\bar{f}_{i j}^{\lambda}\left(z_{j}\right)\right. \\
& -\sum_{\gamma=1}^{n}\left(\partial \bar{f}_{i j}^{\lambda} / \partial z_{j}^{\gamma}\right)\left(z_{j}\right)\left(\left(g_{j}^{\mu-1}(s)\right)^{\gamma}-\left(g_{j}^{0}\right)^{\gamma}\right) \\
& \left.-\sum_{\alpha=1}^{r}\left(\partial f_{i j}^{\lambda} / \partial s_{\alpha}\right)\left(z_{j}, 0\right) s_{\alpha}\right\} \partial / \partial z_{i}^{\lambda} .
\end{aligned}
$$

From (3.vi) and (3.5) $)_{\mu-1}$, we have

$$
\begin{aligned}
\left\|\kappa_{s}^{\mu}\left(\sigma_{i j}^{\mu}(s)\right)\right\|_{(m)}^{\prime} & \ll\left(b_{0} / c_{0}\right) \sum_{v=2}^{\infty} c_{0}^{v}\left(c_{m}^{\prime}\right)^{v}(n+r)^{v} A(s)^{v} \\
& \ll b_{0} c_{m}^{\prime}(n+r) \sum_{v=1}^{\infty}\left\{c_{0} c_{m}^{\prime}(n+r) b / c\right\}^{v} A(s)
\end{aligned}
$$

where $c_{m}^{\prime}$ is the constant introduced in Section 1 . Hence if $c_{0} c_{m}^{\prime}(n+r) b / c<1 / 2$ is satisfied,

$$
b_{0} c_{m}^{\prime}(n+r) \sum_{v=1}^{\infty}\left\{c_{0} c_{m}^{\prime}(n+r) b / c\right\}^{v} A(s) \ll 2 b_{0} c_{0}\left(c_{m}^{\prime}\right)^{2}(n+r)^{2}(b / c) A(s) .
$$

(2) From the definition of $g_{i \mid \mu}^{\prime}(s)$ and (1), we infer (2).

(3) From the definition of $\omega_{i}^{\mu}(s)$,

$$
\bar{\partial}_{b}\left(g_{i \mid u}^{\prime}(s)\right)^{\lambda}+\kappa_{s}^{\mu}\left(\omega_{i}^{\mu}(s)\right)^{\lambda}-\kappa_{s}^{\mu}\left\{\phi\left(\tau^{\mu-1}(s)\right)\left(g_{i}^{\mu-1}(s)-g_{i}^{0}\right)^{\lambda}\right\}=0 .
$$

Then, from $(3.5)_{\mu-1},(3.6)_{\mu-1}$ and (2), we have

$$
\left\|\kappa_{s}^{\mu}\left(\omega_{i}^{\mu}(s)\right)\right\|_{(m)} \ll\left(n b_{0} c_{m} / c_{0}\right)\left\{\sum_{v=1}^{\infty} c_{0}^{v} q^{v} c_{m}^{\nu-1} A(s)^{\nu}\right\} A(s)+\left(d_{m} b / c\right) K_{2} A(s),
$$

where $c_{m}$ and $d_{m}$ are constants introduced in Section 1 . Hence, if $c_{0} q c_{m} b / c<1 / 2$, we have

$$
\left\|\kappa_{s}^{\mu}\left(\omega_{i}^{\mu}(s)\right)\right\|_{(m)} \ll(b / c)\left(2 n b_{0} c_{m} q+d_{m} K_{2}\right) A(s) .
$$

(4) Since $\kappa_{s}^{\mu}\left(\phi\left(\tau^{\mu-1}(s)\right)\right)=\kappa_{s}^{\mu}\left(\phi\left(\tau^{\mu-1}(s)\right)-\phi_{1} \tau^{\mu-1}(s)\right)$,

$$
\begin{aligned}
\left\|\kappa_{s}^{\mu}\left(\phi\left(\tau^{\mu-1}(s)\right)\right)\right\|_{(m)} & \left.\left.\ll\left(b_{0} / c_{0}\right) \sum_{v=2}^{\infty} \mathrm{c}_{0}^{v} q^{v} A(s)^{v}, \quad \text { (by (3.v) and (3.6) }\right)_{\mu-1}\right), \\
& \ll b_{0} q \sum_{v=1}^{\infty}\left(c_{0} q b / c\right)^{v} A(s) .
\end{aligned}
$$

Hence, if $q c_{0} b / c<1 / 2$ is satisfied, we have

$$
\left\|\kappa_{s}^{\mu}\left(\phi\left(\tau^{\mu-1}(s)\right)\right)\right\|_{(m)} \ll 2 b_{0} c_{0} q^{2}(b / c) A(s) .
$$

Since $\xi_{i}^{\mu}(s) \equiv \omega_{i}^{\mu}(s)-\phi\left(\tau^{\mu-1}(s)\right)_{\mid U_{i}} \bmod \left(s^{\mu+1}\right)$, we infer (4) from (3) and this estimate.

Q.E.D.

Using this lemma we shall show (3.5) $)_{\mu}$ and (3.6) ${ }_{\mu}$.

$$
\begin{aligned}
\left|\tau_{\mu}(s)\right| & =\left|\mathscr{L} \boldsymbol{H}_{T^{\prime}}^{1}\left(\sum_{i \in \Lambda} \rho_{i} \kappa_{s}^{\mu}\left(\xi_{i}^{\mu}(s)\right)\right)\right| \\
& \ll c_{2} c_{m} \sum_{i \in \Lambda}\left\|\rho_{i}\right\|_{(m)}\left\|\kappa_{s}^{\mu}\left(\xi_{i}^{\mu}(s)\right)\right\|_{(m)} \\
& \left.\ll(b / c) c_{2} c_{m} K_{4} \sum_{i \in \Lambda}\left\|\rho_{i}\right\|_{(m)} A(s), \quad \text { (by Lemma } 3.10(4)\right),
\end{aligned}
$$


where $c_{2}$ is a constant satisfying $\left|\mathscr{L} \boldsymbol{H}_{T}^{1}, \phi\right| \leqq c_{2}\|\phi\|_{(m)}$ for any $\phi \in \Gamma(M$, $\left.T^{\prime} \otimes\left({ }^{\circ} T^{\prime \prime}\right)^{*}\right)$.

$$
\begin{aligned}
\left\|g_{\mu}^{\prime}(s)\right\|_{(m)}^{\prime} & =\left\|\bar{\partial}_{T^{\prime}}^{(0) *} N_{T^{\prime}}\left(\sum_{i \in \Lambda} \rho_{i} \kappa_{s}^{\mu}\left(\xi_{i}^{\mu}(s)\right)-\phi_{1} \tau_{\mu}(s)\right)\right\|_{(m)}^{\prime} \\
& \ll c_{3}\left\|\sum_{i \in \Lambda} \rho_{i} \kappa_{s}^{\mu}\left(\xi_{i}^{\mu}(s)\right)\right\|_{(m)}+c_{3} c_{4}\left|\tau_{\mu}(s)\right| \\
& \ll(b / c)\left(1+c_{2} c_{4}\right) c_{3} c_{m} K_{4} \sum_{i \in \Lambda}\left\|\rho_{i}\right\|_{(m)} A(s),
\end{aligned}
$$

where $c_{3}$ and $c_{4}$ are constants satisfying

$$
\left\|\bar{\partial}_{T^{\prime}}^{(0) *} N_{T^{\prime}} \phi\right\|_{(m)}^{\prime} \leqq c_{3}\|\phi\|_{(m)} \quad \text { for } \quad \phi \in \Gamma\left(M, T^{\prime} \otimes\left({ }^{o} T^{\prime \prime}\right)^{*}\right)
$$

(cf. [2], Proposition 3.11) and

$$
\|\eta\|_{(m)} \leqq c_{4}|\eta| \quad \text { for any } \quad \eta \in \mathscr{H}
$$

(since $\mathscr{H}$ is finite dimensional), respectively.

Hence if we choose $b$ and $c$ in such a way that $(3.5)_{1},(3.6)_{1},(1)-(4)$ in Lemma 3.10, $(b / c) c_{2} c_{m} K_{4} \sum_{i \in \Lambda}\left\|\rho_{i}\right\|_{(m)}<1,(b / c) K_{2}<1 / 2,(b / c)\left(1+c_{2} c_{4}\right) c_{3} c_{m} K_{4}$ $\times \sum_{i \in \Lambda}\left\|\rho_{i}\right\|_{(m)}<1 / 2$ are all satisfied, (3.5) $)_{\mu}$ and (3.6) $)_{\mu}$ follow.

From these arguments, we infer that $g_{i}(s)$ and $\tau(s)$ are a $\Gamma_{(m)}^{\prime}\left(U_{i}, T^{\prime}\right)$ valued and a $\mathscr{H}$-valued holomorphic functions on some neighbourhood $D^{\prime}$ of 0 in $D$, respectively. Since $m \geqq n+2$, by the Sobolev's lemma, $\left\{g_{i}(s)\right\}_{i \in \Lambda}$ are of class $C^{2}$.

From $(3.1)_{\mu}-(3.3)_{\mu}$, using the same argument at the end of Section 2, we infer that $F=\left(\left\{g_{i}(s)\right\}_{i \in \Lambda}, \tau(s)\right)$ satisfies (3.1), (3.2) and (3.3) for some neighbourhood $D^{\prime}$ of 0 in $D$.

\section{References}

[1] Akahori, T., Intrinsic formula for Kuranishi's $\hat{\partial}_{b}^{\varphi}$, Publ. RIMS, Kyoto Univ., 14 (1978), 615-641.

[2] - Complex analytic construction of the Kuranishi family on a normal strongly pseudo convex manifold, Publ. RIMS, Kyoto Univ., 14 (1978), 789-847.

[3] Folland, G. B. and Kohn, J. J., The Neumann problem for the Cauchy-Riemann complex, Ann. Math. Studies, 75, Princeton Univ. Press, 1972.

[4] Kuranishi, M., Deformations of isolated singularities and $\bar{\partial}_{b}$, Preprint, Columbia Univ., 1973.

[5] Miyajima, K., On the existence of Kuranishi family for deformations of holomorphic maps, Sci. Rep. Kagoshima Univ., 27 (1978), 43-76.

[6] Tanaka, N., A differential geometric study on strongly pseudo-convex manifolds, Lectures in Math., Dep. of Math., Kyoto Univ., 9, 1975. 